\title{
CONSTRUCTING EMBEDDED LATTICE RULES FOR MULTIVARIATE INTEGRATION
}

\author{
RONALD COOLS ${ }^{\dagger}$, FRANCES Y. KUO ${ }^{\ddagger}$, AND DIRK NUYENS ${ }^{\dagger} \S$
}

\begin{abstract}
Lattice rules are a family of equal-weight cubature formulas for approximating highdimensional integrals. By now it is well established that good generating vectors for lattice rules having $n$ points can be constructed component-by-component for integrands belonging to certain weighted function spaces, and that they can achieve the optimal rate of convergence. Although the lattice rules constructed this way are extensible in dimension, they are not extensible in $n$, thus when $n$ is changed the generating vector needs to be constructed anew. In this paper we introduce a new algorithm for constructing good generating vectors for embedded lattice rules which can be used for a range of $n$ while still being extensible in dimension. By using an adaptation of the fast component-by-component construction algorithm (which makes use of fast Fourier transforms), we are able to obtain good generating vectors for thousands of dimensions and millions of points, under both product weight and order-dependent weight settings, at the cost of $\mathcal{O}\left(\operatorname{dn}(\log (n))^{2}\right)$ operations. With a sufficiently large number of points and good overall quality, these embedded lattice rules can be used for practical purposes in the same way as a low-discrepancy sequence. We show for a range of weight settings in an unanchored Sobolev space that our embedded lattice rules achieve the same (optimal) rate of convergence $\mathcal{O}\left(n^{-1+\delta}\right), \delta>0$, as those constructed for a fixed number of points, and that the implied constant only gains a factor of 1.30 to 1.55 .
\end{abstract}

Key words. Numerical integration, Quasi-Monte Carlo methods, Rank-1 lattice rules, Fast component-by-component construction, Embedded lattice rules, Extensible lattice sequences

AMS subject classifications. 65D30, 65D32, 68W40

1. Introduction. Integrals with hundreds or even thousands of dimensions occur in many practical applications in areas such as statistics and mathematical finance. After some appropriate transformations, these integrals can be formulated over the $d$-dimensional unit cube $[0,1]^{d}$ :

$$
I_{d}(f)=\int_{0}^{1} \cdots \int_{0}^{1} f\left(x_{1}, \ldots, x_{d}\right) \mathrm{d} x_{1} \cdots \mathrm{d} x_{d}=\int_{[0,1]^{d}} f(\boldsymbol{x}) \mathrm{d} \boldsymbol{x}
$$

where $f$ is a function of $d$ variables and $\boldsymbol{x}=\left(x_{1}, \ldots, x_{d}\right)$. In this paper we develop an algorithm for constructing "embedded lattice rules" for evaluating such integrals, in the mathematical setting of "weighted reproducing kernel Hilbert spaces". The details about our methods and our function space settings will be discussed in the next section. Here in the Introduction we shall give a general overview of the historical background relevant to the new construction.

When $d$ is large, it is too costly to approximate these integrals using product rules based on low-dimensional integration formulae. The Monte Carlo method is an equal-weight cubature rule of the form

$$
Q_{n, d}(f)=\frac{1}{n} \sum_{k=0}^{n-1} f\left(\boldsymbol{t}_{k}\right),
$$

\footnotetext{
${ }^{\dagger}$ Dept. of Computer Science, K.U.Leuven, Heverlee, Belgium (ronald.cools@cs.kuleuven.be, dirk.nuyens@cs.kuleuven. be)

${ }^{\ddagger}$ School of Mathematics and Statistics, University of New South Wales, Sydney NSW 2052, Australia (f.kuo@unsw.edu.au)

$\S$ Corresponding author.
} 
where the $n$ cubature points $\boldsymbol{t}_{0}, \ldots, \boldsymbol{t}_{n-1}$ are independent and identically distributed uniform random samples from $[0,1]^{d}$. The expected error for the Monte Carlo method is $\sigma(f) / \sqrt{n}$, where $\sigma^{2}(f)$ is the variance of $f$. This leads to a rate of convergence of $\mathcal{O}\left(n^{-1 / 2}\right)$ and the convergence is independent of the dimension $d$ provided that $f \in L_{2}\left([0,1]^{d}\right)$. Quasi-Monte Carlo $(Q M C)$ methods have the same form as the Monte Carlo method, but the cubature points $\boldsymbol{t}_{0}, \ldots, \boldsymbol{t}_{n-1}$, instead of being generated randomly, are chosen deterministically, in the hope of obtaining a better rate of convergence than the Monte Carlo order $\mathcal{O}\left(n^{-1 / 2}\right)$. QMC error bounds are typically in the form of a Koksma-Hlawka-type inequality

$$
\left|I_{d}(f)-Q_{n, d}(f)\right| \leq D\left(\boldsymbol{t}_{0}, \ldots, \boldsymbol{t}_{n-1}\right) V(f),
$$

where $D\left(\boldsymbol{t}_{0}, \ldots, \boldsymbol{t}_{n-1}\right)$ is the discrepancy which measures the non-uniformity of the point set, and $V(f)$ measures the variability of $f$. Error bounds of this form separate the dependence on the cubature points from the dependence on the integrand. In general we do not have control over the integrand and so we focus on choosing cubature points so that the discrepancy is as small as possible. QMC point sets with discrepancy of order $\mathcal{O}\left(n^{-1}(\log n)^{d}\right)$ or better are collectively known as low-discrepancy point sets (see e.g. [12]). This includes, for example, the well-known Sobol' sequences and Niederreiter sequences. Note that the word "sequences" used in this context implies a special feature: the construction of the point set is independent of the number of points $n$ and the dimension $d$. Thus these low-discrepancy sequences are "extensible" in the sense that if we need more cubature points or if we need to increase the dimension, we only need to compute the additional points or the additional components of the points.

Lattice rules are a family of QMC rules which were traditionally used to approximate integrals of periodic functions. The oldest and simplest form of lattice rules, now known as rank-1 lattice rules, have the point set

$$
\left\{\left\{\frac{k \boldsymbol{z}}{n}\right\}: k=0,1, \ldots, n-1\right\} .
$$

Here $\boldsymbol{z}=\left(z_{1}, \ldots, z_{d}\right)$, known as the generating vector, is a $d$-dimensional integer vector having no factor in common with $n$, and the braces around a vector indicate that each component of the vector is to be replaced by its fractional part. Lattice point sets are low-discrepancy point sets although their constructions are typically not extensible. For a long time the most popular way to construct lattice rules in higher dimensions was to take the generating vector of the Korobov form:

$$
\boldsymbol{z}=\left(1, a, a^{2}, \ldots, a^{d-1}\right) \bmod n, \quad 1 \leq a \leq n-1, \operatorname{gcd}(a, n)=1 .
$$

For a given value of $n$ and a given value of $d$, an optimal value of $a$ can be chosen by minimizing some discrepancy measure. However, this value of $a$ is only good for the specific pair of $n$ and $d$, and so if either $n$ or $d$ is changed, we need to obtain a new value of $a$. (One could use a chosen value of $a$ for higher dimensions simply by taking higher powers of $a$ modulo $n$, in which case the projections of the points over consecutive dimensions will remain stationary. Unfortunately, this does not guarantee that the discrepancy measure which we are interested in will remain small.)

In 2002 Sloan and his collaborators (see e.g. [16]) developed the "component-bycomponent (CBC)" construction: the components of $\boldsymbol{z}$ are constructed one at a time to minimize the worst-case error in some weighted function space. The details about 
worst-case analysis and weighted function spaces will be given in the next section. For now we can think of the worst-case error as some form of discrepancy which measures the quality of our lattice rule. Let $e_{n, d}(\boldsymbol{z})=e_{n, d}\left(z_{1}, \ldots, z_{d}\right)$ denote the worst-case error for a rank-1 lattice rule with generating vector $\boldsymbol{z}$. The CBC algorithm goes like this:

Algorithm 1 (CBC Algorithm). Given n,

1. Set $z_{1}=1$.

2. For each $d=2,3, \ldots$, with $z_{1}, \ldots, z_{d-1}$ fixed, choose $z_{d}$ from the set $\{1 \leq z \leq n-1: \operatorname{gcd}(z, n)=1\}$

to minimize the worst-case error $e_{n, d}\left(z_{1}, \ldots, z_{d-1}, z_{d}\right)$.

This construction is extensible in $d$ in the sense that if further dimensions are needed, the algorithm can be restarted in an obvious way. It is, however, not extensible in $n$ and so a different value of $n$ means that a completely new $\boldsymbol{z}$ must be constructed.

It has been proved by Hickernell and Niederreiter in 2003 (see [8]) that good generating vectors for extensible lattice sequences do exist, that is, there exists some vector $\boldsymbol{z}$ which is good for all $n$ and all $d$. However their proof is non-constructive and it leaves no clues as to how to find such a vector $\boldsymbol{z}$. Since $n$ can be arbitrarily large, the components of the generating vector $\boldsymbol{z}$ can also be arbitrarily large, making it rather difficult to find one generating vector that is good simultaneously for all values of $n$. Back in 2000, Hickernell and his collaborators (see [7]) had already experimented with the construction of extensible lattice sequences in an empirical setting. By assuming that

$$
n=b^{m}, \quad b \geq 2, \quad m_{1} \leq m \leq m_{2}, \quad \text { and } \quad 1 \leq d \leq d_{\max },
$$

they designed an algorithm which finds a generating vector of the Korobov form (1.2), with the parameter $a$ chosen by minimizing a loss function

$$
G_{m_{1}, m_{2}, d_{\max }}(a):=\max _{\substack{m_{1} \leq m \leq m_{2} \\ 1 \leq d \leq d_{\max }}} \frac{D_{b^{m}, d}(a)}{D_{b^{m}, d}^{(\text {asy })}} .
$$

Here $D_{b^{m}, d}(a)$ is some discrepancy measure for a $d$-dimensional $b^{m}$-point lattice rule with Korobov parameter $a$, and $D_{b^{m}, d}^{(\text {asy })}$ is some asymptotically derived normalization chosen to ensure that for various values of $m$ and $d$ the discrepancy $D_{b^{m}, d}(a)$ is scaled appropriately for comparison.

Lattice rules constructed in [7] are not so much "extensible" as "embedded" in their nature: the distinction being that extensible rules would leave $n$ unbounded whilst embedded rules have $n$ bounded. Therefore, with each $d$-dimensional generating vector $\boldsymbol{z}$, we have a sequence of point sets

$$
\left\{L_{m}\right\}_{m_{1} \leq m \leq m_{2}},
$$

where each $L_{m}$ is the point set for a lattice rule with $b^{m}$ points, and all the points in $L_{m}$ are also points of $L_{m+1}$. For example, when $b=2$, the number of points doubles as we move along the sequence, and each rule in the sequence contains all the points from the previous rule together with an equal number of new points. This embedding property is very attractive in practice because not only can we easily increase the number of points without wasting any function evaluations, but also we can estimate the error based on the results from successive rules in the sequence. 
Finally we come to the point where we can discuss our new construction. Our algorithm is similar to that of [7], but rather than making use of the Korobov form, our algorithm is based on CBC constructions and so there is no need to assume that $d$ is bounded. For each $m$ from $m_{1}$ to $m_{2}$, we use Algorithm 1 to find a generating vector $\boldsymbol{z}^{(m)}$ for a $b^{m}$-point rule and we store the resulting worst-case error $e_{b^{m}, d}\left(\boldsymbol{z}^{(m)}\right)$ for each $d=1,2, \ldots$. We define a new error measure for our embedded lattice rule by

$$
X_{m_{1}, m_{2}, d}(\boldsymbol{z}):=\max _{m_{1} \leq m \leq m_{2}} \frac{e_{b^{m}, d}(\boldsymbol{z})}{e_{b^{m}, d}\left(\boldsymbol{z}^{(m)}\right)},
$$

where we use $e_{b^{m}, d}\left(\boldsymbol{z}^{(m)}\right)$ as our reference error to provide a good normalization. Our new algorithm goes like this:

Algorithm 2 (CBC Algorithm for Embedded Lattice Rules). Given b, $m_{1}$, $m_{2}$, and the pre-computed values of $e_{b^{m}, d}\left(\boldsymbol{z}^{(m)}\right)$ for each $m_{1} \leq m \leq m_{2}$ and each $d=1,2, \ldots$,

1. Set $z_{1}=1$.

2. For each $d=2,3, \ldots$, with $z_{1}, \ldots, z_{d-1}$ fixed, choose $z_{d}$ from the set $\left\{1 \leq z \leq b^{m_{2}}-1: \operatorname{gcd}(z, b)=1\right\}$

to minimize the error measure $X_{m_{1}, m_{2}, d}\left(z_{1}, \ldots, z_{d-1}, z_{d}\right)$.

Just as is the case for the algorithm in [7], our new algorithm does not come with an a priori error bound, nor does it give us a fully extensible lattice sequence that can be used for arbitrarily large $n$. Having said that, in addition to the bonus that $d$ need not be bounded, our algorithm does have two advantages over the algorithm of [7]. Firstly, it is proved in [10] and in [4] that the CBC algorithm can achieve a rate of convergence of $\mathcal{O}\left(n^{-1+\delta}\right), \delta>0$, if we "randomly shift" the lattice points (see below). Thus the worst-case error $e_{b^{m}, d}\left(\boldsymbol{z}^{(m)}\right)$ can be said to be almost optimal, which means that we are using a fairly reliable normalization. The quantity $X_{m_{1}, m_{2}, d}(\boldsymbol{z})$ represents how much worse our chosen vector $\boldsymbol{z}$ is compared with the vectors $\boldsymbol{z}^{(m)}$ which are the near-optimal choices for different $n=b^{m}$. With all the benefits of having embedded rules, we would be happy with a value of $X_{m_{1}, m_{2}, d}(\boldsymbol{z})$ that is no more than 2 , and this indeed turns out to be the case in all our computations. Thus in practice our construction comes with an a posteriori worst-case error bound that is of optimal order, and within a factor of 2 of that achieved by the original CBC algorithm.

The second advantage of our algorithm is that, surprisingly, it has a relatively low computational cost. Nuyens and Cools $[13,14]$ developed a fast implementation of the CBC algorithm by making use of fast Fourier transforms, which allows the construction with $n$ in the millions and $d$ in the thousands within a feasible time frame. Thus the pre-computation of $\boldsymbol{z}^{(m)}$ for each $m$ can be done in a fast way. In fact if we take $b$ to be prime, our new algorithm based on minimizing $X_{m_{1}, m_{2}, d}(\boldsymbol{z})$ can also be done in a fast way, and the cost depends only on $m_{2}$ rather than the sum of all $m$. The total computational cost of Algorithm 2, including the pre-computations of Algorithm 1, is $\mathcal{O}\left(d n(\log (n))^{2}\right)$ operations.

The description above was a bit simplified: it is actually "randomly-shifted" rank1 lattice rules that we construct generating vectors for. Shifted rank-1 lattice rules are $\mathrm{QMC}$ rules with the point set

$$
\left\{\left\{\frac{k \boldsymbol{z}}{n}+\boldsymbol{\Delta}\right\}: k=0,1, \ldots, n-1\right\},
$$

where $\boldsymbol{z}$ is the generating vector as in a rank-1 lattice rule, and $\boldsymbol{\Delta} \in[0,1]^{d}$ is the shift. In particular, we are interested in using random shifts. There are several advantages 
in doing this: we only need to determine the generating vector; the error expression is simpler (see §2); and most importantly, we can obtain a probabilistic error estimate (see $\S 5)$.

The paper is organized as follows. In $\S 2$ we discuss weighted reproducing kernel Hilbert spaces. We consider weighted Korobov spaces and weighted Sobolev spaces, as it is already known that the CBC algorithm leads to lattice rules which achieve the optimal rate of convergence in these spaces, under suitable conditions on the weights. In $\S 3$ we outline some important properties of embedded lattice rules. In $\S 4$ we discuss the implementation of our new algorithm under two different weight settings: the product weights and the order-dependent weights. We consider lattice rules with $n=b^{m}$ points, and for simplicity we consider only prime base $b$. In $\S 5$ we provide some notes on how to use these embedded lattice rules as a sequence and how to obtain practical error estimation by using random shifts. In $\S 6$ we carry out some numerical experiments to see how well our new lattice rules perform for some finance problems. Finally a brief summary is given in $\S 7$. In the Appendix we list one generating vector up to 360 dimensions (obtained with $b=2, m_{1}=10$ and $m_{2}=20$ ) which is good for $n$ ranging from 1024 to 1048576 .

2. Function space setting. In this section we briefly review the mechanics behind worst-case analysis in a reproducing kernel Hilbert space and the concept of weighted function spaces. For more details, see e.g. $[6,17,5]$.

2.1. Worst-case analysis in reproducing kernel Hilbert spaces. Suppose that our integrands belong to some reproducing kernel Hilbert space $H$. A reproducing kernel $K(\boldsymbol{x}, \boldsymbol{y})$ is a symmetric and positive definite function satisfying $K(\boldsymbol{x}, \cdot) \in H$ for all $\boldsymbol{x} \in[0,1]^{d}$, and $\langle K(\boldsymbol{x}, \cdot), f\rangle_{H}=f(\boldsymbol{x})$ for all $f \in H$ and all $\boldsymbol{x} \in[0,1]^{d}$. The existence of a reproducing kernel follows from the Riesz representation theorem, under the assumption that point evaluations are bounded linear functionals in $H$. We assume that the function $h(\boldsymbol{x}):=\int_{[0,1]^{d}} K(\boldsymbol{x}, \boldsymbol{y}) \mathrm{d} \boldsymbol{y}$ belongs to the space $H$. Then multivariate integration $I_{d}$ is a well-defined continuous linear functional, with $h$ as its representer in $H$, and the initial error for multivariate integration is

$$
\left\|I_{d}\right\|=\|h\|_{H}=\left(\int_{[0,1]^{2 d}} K(\boldsymbol{x}, \boldsymbol{y}) \mathrm{d} \boldsymbol{x} \mathrm{d} \boldsymbol{y}\right)^{1 / 2} .
$$

To ensure that $H$ is embedded in $L_{2}\left([0,1]^{d}\right)$, we assume that $\int_{[0,1]^{d}} K(\boldsymbol{x}, \boldsymbol{x}) \mathrm{d} \boldsymbol{x}<\infty$ which also guarantees that the initial error is finite.

The worst-case error for a QMC rule $Q_{n, d}$ with point set $P_{n}=\left\{\boldsymbol{t}_{0}, \ldots, \boldsymbol{t}_{n-1}\right\}$ in $H$ is defined by

$$
e_{n, d}\left(P_{n}, K\right):=\sup _{f \in H,\|f\|_{H} \leq 1}\left|I_{d}(f)-Q_{n, d}(f)\right| .
$$

Clearly $\left|I_{d}(f)-Q_{n, d}(f)\right| \leq e_{n, d}\left(P_{n}, K\right)\|f\|_{H}$. Thus the worst-case error can be thought of as a type of discrepancy measure (see (1.1)). Using the reproducing property of $K$ one can derive an expression for the squared worst-case error

$$
\begin{aligned}
& e_{n, d}^{2}\left(P_{n}, K\right) \\
& \quad=\int_{[0,1]^{2 d}} K(\boldsymbol{x}, \boldsymbol{y}) \mathrm{d} \boldsymbol{x} \mathrm{d} \boldsymbol{y}-\frac{2}{n} \sum_{k=0}^{n-1} \int_{[0,1]^{d}} K\left(\boldsymbol{t}_{k}, \boldsymbol{x}\right) \mathrm{d} \boldsymbol{x}+\frac{1}{n^{2}} \sum_{k=0}^{n-1} \sum_{\ell=0}^{n-1} K\left(\boldsymbol{t}_{k}, \boldsymbol{t}_{\ell}\right) .
\end{aligned}
$$


This explicit expression is especially useful when the reproducing kernel has a simple closed form.

The error expression is simpler for shift-invariant kernels. A shift-invariant kernel is one which satisfies

$$
K(\{\boldsymbol{x}+\boldsymbol{\Delta}\},\{\boldsymbol{y}+\boldsymbol{\Delta}\})=K(\boldsymbol{x}, \boldsymbol{y})=K(\{\boldsymbol{x}-\boldsymbol{y}\}, \mathbf{0}) \quad \forall \boldsymbol{x}, \boldsymbol{y}, \boldsymbol{\Delta} \in[0,1]^{d} .
$$

For any arbitrary kernel $K$, there is an associated shift-invariant kernel $K^{\text {sh }}$ defined by

$$
K^{\mathrm{sh}}(\boldsymbol{x}, \boldsymbol{y}):=\int_{[0,1]^{d}} K(\{\boldsymbol{x}+\boldsymbol{\Delta}\},\{\boldsymbol{y}+\boldsymbol{\Delta}\}) \mathrm{d} \boldsymbol{\Delta} .
$$

Let $P_{n}+\boldsymbol{\Delta}:=\left\{\{\boldsymbol{t}+\boldsymbol{\Delta}\}: \boldsymbol{t} \in P_{n}\right\}$ denote the point set obtained by adding a shift $\boldsymbol{\Delta}$ to every point in $P_{n}$ and then taking the fractional part of every component. One can easily derive the important property that

$$
\begin{aligned}
e_{n, d}^{2}\left(P_{n}, K^{\mathrm{sh}}\right) & =\int_{[0,1]^{d}} e_{n, d}^{2}\left(P_{n}+\boldsymbol{\Delta}, K\right) \mathrm{d} \boldsymbol{\Delta} \\
& =-\int_{[0,1]^{d}} K^{\mathrm{sh}}(\boldsymbol{x}, \mathbf{0}) \mathrm{d} \boldsymbol{x}+\frac{1}{n^{2}} \sum_{k=0}^{n-1} \sum_{\ell=0}^{n-1} K^{\mathrm{sh}}\left(\left\{\boldsymbol{t}_{k}-\boldsymbol{t}_{\ell}\right\}, \mathbf{0}\right)
\end{aligned}
$$

We want to use randomly-shifted rank-1 lattice rules in the function space with kernel $K$. It makes sense to study this "shift-averaged" error given by (2.2). In addition, the double sum reduces to a single sum for lattice rules, explicitly we have

$$
e_{n, d}^{2}(\boldsymbol{z})=-\int_{[0,1]^{d}} K^{\mathrm{sh}}(\boldsymbol{x}, \mathbf{0}) \mathrm{d} \boldsymbol{x}+\frac{1}{n} \sum_{k=0}^{n-1} K^{\mathrm{sh}}\left(\left\{\frac{k \boldsymbol{z}}{n}\right\}, \mathbf{0}\right) .
$$

We shall refer to this quantity as the worst-case error for randomly-shifted rank-1 lattice rules, and it is this worst-case error which we shall consider throughout the rest of the paper. Note that $K^{\text {sh }}=K$ if $K$ is already shift-invariant.

2.2. Weighted function spaces. In this paper we consider reproducing kernel Hilbert spaces $H$ with kernels of the form

$$
K(\boldsymbol{x}, \boldsymbol{y})=\sum_{\mathfrak{u} \subseteq\{1, \ldots, d\}} \gamma_{\mathfrak{u}} \prod_{j \in \mathfrak{u}} \eta\left(x_{j}, y_{j}\right)
$$

with $\eta(x, y)=\eta(y, x)$. This general form of kernels has been considered in [17, 5]. The space $H$ is a direct sum of $2^{d}$ reproducing kernel Hilbert spaces $H_{\mathfrak{u}}$, where each $H_{\mathfrak{u}}$ is a $|\mathfrak{u}|$-dimensional tensor-product space with kernel $K_{\mathfrak{u}}\left(\boldsymbol{x}_{u}, \boldsymbol{y}_{u}\right)=\gamma_{\mathfrak{u}} \prod_{j \in \mathfrak{u}} \eta\left(x_{j}, y_{j}\right)$. Functions in $H_{\mathfrak{u}}$ depend only on the set of variables $\boldsymbol{x}_{\mathfrak{u}}:=\left\{x_{j}: j \in \mathfrak{u}\right\}$. Functions $f \in H$ have the natural decomposition

$$
f(\boldsymbol{x})=\sum_{\mathfrak{u} \subseteq\{1, \ldots, d\}} f_{\mathfrak{u}}\left(\boldsymbol{x}_{\mathfrak{u}}\right), \quad \text { with } \quad f_{\mathfrak{u}}\left(\boldsymbol{x}_{\mathfrak{u}}\right)=\left\langle K_{\mathfrak{u}}\left(\boldsymbol{x}_{u}, \cdot\right), f\right\rangle_{H} \quad \text { and } \quad f_{\mathfrak{u}} \in H_{\mathfrak{u}} .
$$

We associate a weight parameter $\gamma_{\mathfrak{u}}$ with each set of variables $\boldsymbol{x}_{\mathfrak{u}}$, which describes the level of interaction between the variables in $\boldsymbol{x}_{\mathfrak{u}}$. The $2^{d}$ weights $\gamma_{\mathfrak{u}}$ together model the relative importance between various sets of variables. Roughly speaking, a small 
$\gamma_{\mathfrak{u}}$ means that the contribution of the term $f_{\mathfrak{u}}$ is small compared with the other terms. To fix the scaling we set $\gamma_{\emptyset}=1$. The limiting case of $\gamma_{\mathfrak{u}}=0$ implies that there is no $f_{\mathfrak{u}}$ term in the decomposition, or in other words, there is no interaction between the variables in $\boldsymbol{x}_{\mathfrak{u}}$.

The worst-case error for randomly-shifted rank-1 lattice rules (see (2.3)) is expressed in terms of the associated shift-invariant kernel

$$
K^{\mathrm{sh}}(\boldsymbol{x}, \boldsymbol{y})=\sum_{\mathfrak{u} \subseteq\{1, \ldots, d\}} \gamma_{\mathfrak{u}} \prod_{j \in \mathfrak{u}} \omega\left(\left\{x_{j}-y_{j}\right\}\right), \quad \text { with } \omega(x)=\int_{0}^{1} \eta(\{x+\Delta\}, \Delta) \mathrm{d} \Delta .
$$

Note that $\omega$ is symmetric, i.e. $\omega(x)=\omega(1-x)$. For simplicity we impose the additional condition $\int_{0}^{1} \omega(x) \mathrm{d} x=0$. The initial error is then 1 and the worst-case error (2.3) then satisfies

$$
e_{n, d}^{2}(\boldsymbol{z})=-1+\frac{1}{n} \sum_{k=0}^{n-1} \sum_{\mathfrak{u} \subseteq\{1, \ldots, d\}} \gamma_{\mathfrak{u}} \prod_{j \in \mathfrak{u}} \omega\left(\left\{\frac{k z_{j}}{n}\right\}\right) .
$$

(If the initial error were not 1 , we could consider instead the relative error, that is, we divide $e_{n, d}^{2}(\boldsymbol{z})$ by the squared initial error. The expression for the relative error would be of the same form as (2.4), but with rescaled weights $\gamma_{\mathfrak{u}}$.)

To have a function space that best describes our integrand $f$, we must choose the weights $\gamma_{\mathfrak{u}}$ according to the dimension structure of $f$. (See [3] for two notions of effective dimension, and see [19] for numerical schemes to estimate them.) When the nominal dimension is $d$, there are $2^{d}$ weights $\gamma_{\mathfrak{u}}$ to decide upon. Here are a few options to help limit the choices:

(i) Product weights: Here $\gamma_{\mathfrak{u}}:=\prod_{j \in \mathfrak{u}} \gamma_{\{j\}}$, that is, the weight associated with the set of variables $\boldsymbol{x}_{\mathfrak{u}}$ is automatically assigned the product of the weights for each individual variable $x_{j}$ in this set. The product-weight setting leads to a tensor product space $H$, and the worst-case error for randomly-shifted rank-1 lattice rules satisfies

$$
e_{n, d}^{2}(\boldsymbol{z})=-1+\frac{1}{n} \sum_{k=0}^{n-1} \prod_{j=1}^{d}\left(1+\gamma_{j} \omega\left(\left\{\frac{k z_{j}}{n}\right\}\right)\right) .
$$

(ii) Order-dependent weights: Here $\gamma_{\mathfrak{u}}:=\Gamma_{|\mathfrak{u}|}$, that is, the weight associated with the set of variables $\boldsymbol{x}_{\mathfrak{u}}$ depends only on the cardinality of $\mathfrak{u}$. In this case the worst-case error for randomly-shifted rank-1 lattice rules can be written as

$$
e_{n, d}^{2}(\boldsymbol{z})=\frac{1}{n} \sum_{k=0}^{n-1} \sum_{\ell=1}^{d} \Gamma_{\ell} \sum_{\substack{\mathfrak{u} \subseteq\{1, \ldots, d\} \\|\mathfrak{u}|=\ell}} \prod_{j \in \mathfrak{u}} \omega\left(\left\{\frac{k z_{j}}{n}\right\}\right) .
$$

(iii) Finite-order weights: Here $\gamma_{\mathfrak{u}}=0$ for all $|\mathfrak{u}|>q$, with some fixed number $q$. The finite-order-weight setting can be very useful because many high-dimensional integrals in practice do appear to have a finite order of just 2 or 3 . We often combine the finite-order-weight setting with the order-dependent-weight setting.

We end this section with two important examples of such spaces.

Example 1 (Weighted Korobov spaces). The weighted Korobov space consists of one-periodic functions with absolutely convergent Fourier series, see e.g. [5]. The 
reproducing kernel is already shift-invariant, with

$$
\eta(x, y)=\omega(\{x-y\}) \quad \text { and } \quad \omega(x)=\sum_{h \in \mathbb{Z} \backslash\{0\}} \frac{e^{2 \pi \mathrm{i} h x}}{|h|^{\alpha}},
$$

where $\alpha>1$ is a smoothness parameter which corresponds to the rate of decay of the Fourier coefficients. It is known that good (unshifted or randomly-shifted) rank-1 lattice rules can be constructed using the $\mathrm{CBC}$ algorithm to achieve the optimal rate of convergence $\mathcal{O}\left(n^{-\alpha / 2+\delta}\right), \delta>0$ (see [10,4]). Note that when $\alpha$ is an even integer, $\omega$ can be written in terms of the Bernoulli polynomial of degree $\alpha$ :

$$
\omega(x)=\frac{(2 \pi)^{\alpha}}{(-1)^{\frac{\alpha}{2}-1} \alpha !} B_{\alpha}(x) .
$$

Example 2 (Weighted Sobolev spaces). The weighted Sobolev space (with smoothness one) consists of functions with square-integrable mixed first derivatives, see e.g. [17]. These functions are in general non-periodic. There are a few variants of these spaces depending on how the inner product is defined. One popular variant, now commonly referred to as the unanchored Sobolev space, corresponds to

$$
\eta(x, y)=\frac{1}{2} B_{2}(\{x-y\})+\left(x-\frac{1}{2}\right)\left(y-\frac{1}{2}\right) \text { and } \omega(x)=B_{2}(x)=x^{2}-x+\frac{1}{6},
$$

which satisfies the condition $\int_{0}^{1} \omega(x) \mathrm{d} x=0$. This space has a direct association with the ANOVA decomposition of functions (see e.g. [3, 19]). It is known that good randomly-shifted rank-1 lattice rules can be constructed using the $\mathrm{CBC}$ algorithm to achieve the optimal rate of convergence $\mathcal{O}\left(n^{-1+\delta}\right), \delta>0$, in a probabilistic sense (see $[10,4])$.

3. Properties of embedded lattice rules. Before we get into the ideas behind the fast implementations of CBC algorithms, we need to review some fundamental properties of embedded lattice rules.

Given an integer base $b \geq 2$, a fixed dimension $d$, and any integer vector $\boldsymbol{z} \in \mathbb{Z}^{d}$ whose components are all co-prime with $b$, define

$$
L_{m}=L_{m}(\boldsymbol{z}):=\left\{\left\{\frac{k \boldsymbol{z}}{b^{m}}\right\}: k=0,1, \ldots, b^{m}-1\right\}, \quad m=0,1, \ldots
$$

The following properties hold for all $m \geq 0$.

Property 1. Clearly $L_{m}$ is a point set for a rank-1 lattice rule with $b^{m}$ distinct points.

Property 2. The generating vector for the lattice point set $L_{m}$ can be reduced modulo $b^{m}$ to give the same point set, i.e., $L_{m}(\boldsymbol{z})=L_{m}\left(\boldsymbol{z} \bmod b^{m}\right)$.

Property 3. The lattice point set $L_{m}$ is embedded in $L_{m+1}$, i.e., $L_{m} \subset L_{m+1}$.

PROPERTY 4 . The points in $L_{m}$ are exactly those points in $L_{m+1}$ whose indices $k$ in (3.1) are multiples of $b$.

For a given $m$, we can construct a generating vector $z$ using Algorithm 1 with $n=b^{m}$ so that $L_{m}$ is a "good" lattice point set with $b^{m}$ points. Due to Property 2, we can restrict our search for the components of $\boldsymbol{z}$ to a finite set as indicated in Algorithm 1, with the largest possible choice being $b^{m}-1$. Note that the goodness of the point set $L_{m}$ in no way implies that all smaller lattice point sets embedded in $L_{m}$ are good. On the contrary, it is quite possible that the same generating vector $\boldsymbol{z}$ leads to a very poor point set $L_{\ell}$ for $\ell<m$ (as will be demonstrated in $\S 6.1$ ). 
This brings us to Algorithm 2, where a generating vector $\boldsymbol{z}$ is constructed with the hope that the lattice point sets $L_{m}$, for all values of $m$ in a finite range $m_{1} \leq m \leq m_{2}$, would be good simultaneously. The assumption that $m$ is bounded from above by $m_{2}$ means that the maximum number of points is $b^{m_{2}}$; hence we can restrict the components of $\boldsymbol{z}$ to be at most $b^{m_{2}}-1$, ensuring a finite search space. The quality of this generating vector $\boldsymbol{z}$ is assessed by the error measure $X_{m_{1}, m_{2}, d}(\boldsymbol{z})$ defined in (1.3), which compares the worst-case error of $\boldsymbol{z}$ with the worst-case error of the near-optimal generating vectors constructed by Algorithm 1 for each $m$ in our range. Put differently, $X_{m_{1}, m_{2}, d}(\boldsymbol{z})$ measures how much worse than optimal our "one for all" vector $\boldsymbol{z}$ is, and Algorithm 2 selects a vector $\boldsymbol{z}$ so that $X_{m_{1}, m_{2}, d}(\boldsymbol{z})$ is as small as possible for $m$ in the prescribed range. This can be described as the "mini-max" optimization of the worst-case error ratios. The quality of $\boldsymbol{z}$ as a "one for all" vector is assured as long as $X_{m_{1}, m_{2}, d}(\boldsymbol{z})$ is uniformly bounded by a small constant, say 2 .

4. Fast construction of embedded lattice rules. Before we can use Algorithm 2, we need to use Algorithm 1 to find the near-optimal generating vectors for each $m$ from $m_{1}$ to $m_{2}$ and store the worst-case errors in each dimension. The apparent cost of Algorithm 1 for $n$ points up to dimension $d$ is $\mathcal{O}\left(d^{2} n^{2}\right)$, and so the cost of the pre-computations for Algorithm 2 up to $n=b^{m_{2}}$ points can be $\mathcal{O}\left(d^{2} n^{2} \log (n)\right)$. Once this is done, the cost of Algorithm 2 appears to be another $\mathcal{O}\left(d^{2} n^{2} \log (n)\right)$. Thus unless we can speed up the computations in some way, Algorithm 2 would be a mere theoretical exercise.

Three ideas are used to obtain a fast alternative for Algorithm 2 (the first two were already known for Algorithm 1):

(i) The nature of the CBC algorithm is iterative with respect to the dimension. Therefore we will store previous calculations, sacrificing memory for speed. This reduces the construction cost of Algorithm 1 to $\mathcal{O}\left(d n^{2}\right)$.

(ii) The calculation of the worst-case errors resembles a structured matrix-vector product which can be computed in a fast way. Thus the construction cost of Algorithm 1 can be dramatically reduced, to $\mathcal{O}(d n \log (n))$, implying that the cost of the pre-computations for Algorithm 2 is $\mathcal{O}\left(\operatorname{dn}(\log (n))^{2}\right)$.

(iii) The calculation of the worst-case error $e_{n, d}(\boldsymbol{z})$ for the maximum number of points $n=b^{m_{2}}$ contains within it all worst-case errors $e_{b^{m}, d}(\boldsymbol{z})$ for $m \leq m_{2}$. This means that, with a slight adaptation of the algorithm, the actual cost of Algorithm 2 is only $\mathcal{O}(d n \log (n))$ while the cost for those pre-computations based on Algorithm 1 is $\mathcal{O}\left(\operatorname{dn}(\log (n))^{2}\right)$. In other words, we get the embedded rule almost for free.

In the next three subsections we will explain these ideas as independently of each other as possible.

4.1. Matrix-vector formulation. Suppose we are at the point in the CBC algorithm where we want to choose the $d$ th component $z_{d}$. It makes sense to consider the squared worst-case error $e_{n, d}^{2}(\boldsymbol{z})=e_{n, d}^{2}\left(z_{1}, \ldots, z_{d}\right)$ as a function of $z_{d}$, and we denote it by $E_{d}^{2}\left(z_{d}\right)$. First we will rewrite the expression for $E_{d}^{2}\left(z_{d}\right)$ in a form that is more suitable for recursion with respect to $d$. For computational efficiency we only consider two types of weights.

(i) Product weights:

$$
E_{d}^{2}\left(z_{d}\right)=-1+\frac{1}{n} \sum_{k=0}^{n-1}\left(1+\gamma_{d} \omega\left(\left\{\frac{k z_{d}}{n}\right\}\right)\right) \underbrace{\prod_{j=1}^{d-1}\left(1+\gamma_{j} \omega\left(\left\{\frac{k z_{j}}{n}\right\}\right)\right)}_{p_{d-1}(k)}
$$




$$
=e_{n, d-1}^{2}\left(z_{1}, \ldots, z_{d-1}\right)+\frac{\gamma_{d}}{n} \sum_{k=0}^{n-1} \omega\left(\left\{\frac{k z_{d}}{n}\right\}\right) p_{d-1}(k),
$$

where $e_{n, 0}^{2}:=0$ and the products $p_{d}(k)$ are defined recursively by

$$
\begin{aligned}
& p_{0}(k):=1, \\
& p_{d}(k):=\left(1+\gamma_{d} \omega\left(\left\{\frac{k z_{d}}{n}\right\}\right)\right) p_{d-1}(k) .
\end{aligned}
$$

(ii) Order-dependent weights (which may be of finite order q):

$$
\begin{aligned}
E_{d}^{2}\left(z_{d}\right)=\frac{1}{n} \sum_{k=0}^{n-1} \sum_{\ell=1}^{\min (q, d)} \Gamma_{\ell}(\underbrace{\sum_{\substack{u \subseteq\{1, \ldots, d-1\} \\
|\mathfrak{u}|=\ell}} \prod_{j \in \mathfrak{u}} \omega\left(\left\{\frac{k z_{j}}{n}\right\}\right)}_{p_{d-1, \ell}(k)} \\
+\omega\left(\left\{\frac{k z_{d}}{n}\right\}\right) \underbrace{\prod_{j \in \mathfrak{u}} \omega\left(\left\{\frac{k z_{j}}{n}\right\}\right)}_{\substack{\mathfrak{u} \subseteq\{1, \ldots, d-1\} \\
|\mathfrak{u}|=\ell-1}}) \\
=e_{n, d-1}^{2}\left(z_{1}, \ldots, z_{d-1}\right)+\frac{1}{n} \sum_{k=0}^{n-1} \omega\left(\left\{\frac{k z_{d-1, \ell-1}(k)}{n}\right\}\right)\left(\sum_{\ell=1}^{\min (q, d)} \Gamma_{\ell} p_{d-1, \ell-1}(k)\right)
\end{aligned}
$$

where $e_{n, 0}^{2}:=0$ and the products $p_{d, \ell}(k)$ are defined recursively by

$$
\begin{aligned}
p_{d, 0}(k) & :=1, \\
p_{d, \ell}(k) & :=p_{d-1, \ell}(k)+\omega\left(\left\{\frac{k z_{d}}{n}\right\}\right) p_{d-1, \ell-1}(k) .
\end{aligned}
$$

Let $\mathbb{Z}_{n}:=\{0,1, \ldots, n-1\}$ denote the set of the integers modulo $n$, and $U_{n}$ the multiplicative group of integers modulo $n$, i.e.,

$$
U_{n}:=\left\{u \in \mathbb{Z}_{n}: \operatorname{gcd}(u, n)=1\right\},
$$

with the cardinality of $U_{n}$ given by the Euler totient function $\phi(n)=\left|U_{n}\right|$. We need to evaluate $E_{d}^{2}\left(z_{d}\right)$ for every choice of $z_{d} \in U_{n}$, which suggests the definition of the vectors

$$
\boldsymbol{E}_{d}^{2}:=\left[E_{d}^{2}(z)\right]_{z \in U_{n}}, \quad \boldsymbol{p}_{d}:=\left[p_{d}(k)\right]_{k \in \mathbb{Z}_{n}}, \quad \boldsymbol{p}_{d, \ell}:=\left[p_{d, \ell}(k)\right]_{k \in \mathbb{Z}_{n}},
$$

and the matrix

$$
\boldsymbol{\Omega}_{n}:=\left[\omega\left(\left\{\frac{k z}{n}\right\}\right)\right]_{\substack{z \in U_{n} \\ k \in \mathbb{Z}_{n}}}=\left[\omega\left(\frac{k z \bmod n}{n}\right)\right]_{\substack{z \in U_{n} \\ k \in \mathbb{Z}_{n}}} .
$$

We now observe from (4.1) and (4.3) that the squared worst-case error vector $\boldsymbol{E}_{d}^{2}$ can be expressed in terms of a matrix-vector product with the matrix $\boldsymbol{\Omega}_{n}$ as

$$
\boldsymbol{E}_{d}^{2}:=\mathbf{1}_{\phi(n)} e_{n, d-1}^{2}\left(z_{1}, \ldots, z_{d-1}\right)+\frac{\gamma_{d}}{n} \boldsymbol{\Omega}_{n} \boldsymbol{p}_{d-1} \quad \text { (product) }
$$


$\boldsymbol{E}_{d}^{2}:=\mathbf{1}_{\phi(n)} e_{n, d-1}^{2}\left(z_{1}, \ldots, z_{d-1}\right)+\frac{1}{n} \boldsymbol{\Omega}_{n}\left(\sum_{\ell=1}^{\min (q, d)} \Gamma_{\ell} \boldsymbol{p}_{d-1, \ell-1}\right) \quad$ (order-dependent)

where $\mathbf{1}_{t}$ denotes a vector of ones of length $t$. Algorithm 1 picks the value of $z_{d} \in U_{n}$ which corresponds to the smallest entry in $\boldsymbol{E}_{d}^{2}$. Then it is clear from (4.2) and (4.4) that the vectors $\boldsymbol{p}_{d}$ or $\boldsymbol{p}_{d, \ell}$ for the next iteration can be obtained recursively via

$$
\begin{aligned}
\boldsymbol{p}_{d} & :=\left(\mathbf{1}_{n}+\gamma_{d} \boldsymbol{\Omega}_{n}\left(z_{d}\right)\right) * \boldsymbol{p}_{d-1} & & \text { (product) } \\
\boldsymbol{p}_{d, \ell} & :=\boldsymbol{p}_{d-1, \ell}+\boldsymbol{\Omega}_{n}\left(z_{d}\right) * \boldsymbol{p}_{d-1, \ell-1} & & \text { (order-dependent) }
\end{aligned}
$$

where $\boldsymbol{\Omega}_{n}\left(z_{d}\right)$ denotes the row of $\boldsymbol{\Omega}_{n}$ corresponding to the chosen $z_{d}$, and the operator * denotes the element-wise vector multiplication. Since the vectors $\boldsymbol{p}_{d-1}$ or $\boldsymbol{p}_{d-1, \ell}$ are no longer needed in the next iteration, we can simply overwrite $\boldsymbol{p}_{d-1}$ with $\boldsymbol{p}_{d}$ or overwrite $\boldsymbol{p}_{d-1, \ell}$ with $\boldsymbol{p}_{d, \ell}$. Hence, starting with the vectors $\boldsymbol{p}_{0}:=\mathbf{1}_{n}$ or $\boldsymbol{p}_{0, \ell}:=\mathbf{1}_{n}$, we require only $\mathcal{O}(n)$ storage for product weights and $\mathcal{O}(q n)$ storage for order-dependent weights of finite order $q$.

The trick now is to order the indices $z \in U_{n}$ and $k \in \mathbb{Z}_{n}$ in (4.5) and (4.6) in a clever way to allow fast matrix-vector multiplications.

4.2. Fast matrix-vector product. To get an idea of the general concept, we first consider the matrix $\boldsymbol{\Omega}_{n}$ for $n$ prime. Using the Rader factorization [15, 18], this matrix can be reordered such that it has a circulant submatrix of size $(n-1) \times(n-1)$. This is achieved by taking the indices as $z=g^{i}$ and $k=g^{-i^{\prime}}$ for $0 \leq i, i^{\prime} \leq n-2$, where $g$ is a primitive root of $n$ (i.e. $g$ is a generator for the cyclic group $U_{n}$ ). We denote this particular ordering of $\boldsymbol{\Omega}_{n}$ by $\boldsymbol{\Omega}_{n}^{\langle g\rangle}$.

The effect of such reordering is explained most easily by an example. Take $n=11$, then the possible components for the generating vector which we want to consider are

$$
U_{11}=\{1,2,3,4,5,6,7,8,9,10\} .
$$

Since $n$ is prime, we can always find a generator for this group. For our example we choose $g=2$. The ordering for the $z$ indices we want to use is then

$$
\left[g^{i}: 0 \leq i \leq 9\right]=[1,2,4,8,5,10,9,7,3,6] .
$$

For the indices $k \neq 0$ we take the negative powers of $g$

$$
\left[g^{-i^{\prime}}: 0 \leq i^{\prime} \leq 9\right]=[1,6,3,7,9,10,5,8,4,2],
$$

which can easily be seen as reversing the order of the $z$ indices, but keeping the first index fixed (the zero-th power of $g$ ). This reordering, together with a column of zeros for $k=0$, gives a matrix with the following structure

$$
\boldsymbol{\Omega}_{11}^{\langle 2\rangle}=\omega\left(\frac{1}{11}\left[\begin{array}{ccccccccccc}
1 & 6 & 3 & 7 & 9 & 10 & 5 & 8 & 4 & 2 & 0 \\
2 & 1 & 6 & 3 & 7 & 9 & 10 & 5 & 8 & 4 & 0 \\
4 & 2 & 1 & 6 & 3 & 7 & 9 & 10 & 5 & 8 & 0 \\
8 & 4 & 2 & 1 & 6 & 3 & 7 & 9 & 10 & 5 & 0 \\
5 & 8 & 4 & 2 & 1 & 6 & 3 & 7 & 9 & 10 & 0 \\
10 & 5 & 8 & 4 & 2 & 1 & 6 & 3 & 7 & 9 & 0 \\
9 & 10 & 5 & 8 & 4 & 2 & 1 & 6 & 3 & 7 & 0 \\
7 & 9 & 10 & 5 & 8 & 4 & 2 & 1 & 6 & 3 & 0 \\
3 & 7 & 9 & 10 & 5 & 8 & 4 & 2 & 1 & 6 & 0 \\
6 & 3 & 7 & 9 & 10 & 5 & 8 & 4 & 2 & 1 & 0
\end{array}\right]\right)
$$


As was proved in [13], we can halve the number of rows and the number of columns of the submatrix $\boldsymbol{C}_{11}$ due to the symmetry $\omega(x)=\omega(1-x)$. More specifically, we can write $\boldsymbol{C}_{11}$ in a reduced form (denoted by a $\sim$ instead of $=$ )

$$
\boldsymbol{\Omega}_{11}^{\langle 2\rangle} \sim \omega\left(\frac{1}{11}\left[\begin{array}{llllll}
1 & 6 & 3 & 7 & 9 & 0 \\
2 & 1 & 6 & 3 & 7 & 0 \\
4 & 2 & 1 & 6 & 3 & 0 \\
8 & 4 & 2 & 1 & 6 & 0 \\
5 & 8 & 4 & 2 & 1 & 0
\end{array}\right]\right),
$$

where we remember that the full matrix $\boldsymbol{\Omega}_{11}^{\langle 2\rangle}$ can be obtained by doubling each partition vertically and also horizontally up to the double bar.

A matrix with a structure like the submatrix $\boldsymbol{C}_{11}$ (as well as $\widetilde{\boldsymbol{C}}_{11}$ ) is called a circulant matrix and is completely defined by its first column $\boldsymbol{c}$. Moreover, matrixvector multiplication with a circulant matrix of order $n$ can be done in $\mathcal{O}(n \log (n))$ using fast Fourier transforms.

LEMma 4.1 (Fast matrix-vector product with a circulant matrix). A matrixvector product with a circulant matrix $\boldsymbol{C}_{n}$ of order $n$, with first column $\boldsymbol{c}$, takes $\mathcal{O}(n \log (n))$ operations instead of the usual $\mathcal{O}\left(n^{2}\right)$, and uses $\mathcal{O}(n)$ memory, when using its similarity transform combined with a fast Fourier algorithm for calculating the discrete Fourier transform $\boldsymbol{F}_{n}$ and its inverse $\boldsymbol{F}_{n}^{-1}$ :

$$
\begin{aligned}
\boldsymbol{C}_{n} \boldsymbol{x} & =\boldsymbol{F}_{n}^{-1} \operatorname{diag}\left(\boldsymbol{F}_{n} \boldsymbol{c}\right) \boldsymbol{F}_{n} \boldsymbol{x} \\
& =\operatorname{IFFT}(\operatorname{FFT}(\boldsymbol{c}) * \operatorname{FFT}(\boldsymbol{x})) .
\end{aligned}
$$

A fast $\mathrm{CBC}$ algorithm based on this fast matrix-vector product was first introduced for $n$ prime in [13] and later extended for composite $n$ in [14]. The general idea for composite $n$ is that the complete matrix $\boldsymbol{\Omega}_{n}$ can be partitioned in blocks which have a circulant or block-circulant structure. Basically, we consider a vertical partition $\boldsymbol{A}_{d_{i}}^{\langle g\rangle}$ for each divisor $d_{i}$ of $n$

$$
\boldsymbol{\Omega}_{n}^{\langle g\rangle}:=\left[\boldsymbol{A}_{d_{1}}^{\langle g\rangle}\left|\boldsymbol{A}_{d_{2}}^{\langle g\rangle}\right| \cdots \mid \boldsymbol{A}_{d_{\nu}}^{\langle g\rangle}\right]
$$

where $g$ is a generator (or technically, a set of generators) for the group $U_{n}$, and each $\boldsymbol{A}_{d_{i}}^{\langle g\rangle}$ is constructed out of circulant (or technically, nested block circulant) matrices $\boldsymbol{B}_{n / d_{i}}^{\langle g\rangle}$. The full details can be found in [14] where it is described how the ordering is done and why a matrix-vector multiplication with $\boldsymbol{\Omega}_{n}^{\langle g\rangle}$ takes $\mathcal{O}(n \log (n))$ time using $\mathcal{O}(n)$ memory.

For embedded lattice rules, we shall restrict ourselves to $n$ being a power of a prime $p$, because in this case the matrix $\boldsymbol{\Omega}_{p^{m}}^{\langle g\rangle}$ is of a particularly simple form. For this case we have $\left|U_{p^{m}}\right|=p^{m-1}(p-1)$. We will state the results for $p \neq 2$ and $p=2$ in two separate theorems below; both results follow from [14, Theorem 4 and Corollary 4]. These two theorems differ in subtle yet crucial ways: the form of the matrices $\boldsymbol{B}_{p^{m}}^{\langle g\rangle}$ differs, and there are two degenerate cases $\boldsymbol{B}_{2^{1}}^{\langle 5\rangle}$ and $\boldsymbol{B}_{2^{0}}^{\langle 5\rangle}$ for $p=2$, while there is only one degenerate case $\boldsymbol{B}_{p^{0}}^{\langle g\rangle}$ for $p \neq 2$. In the theorems $\mathbf{1}_{t} \otimes \boldsymbol{B}$ denotes the stacking of $t$ replications of a matrix $\boldsymbol{B}$.

TheOREM 4.2 (Block partitioning $\boldsymbol{\Omega}_{p^{m}}, p$ prime, $p \neq 2$ ). The matrix

$$
\boldsymbol{\Omega}_{p^{m}}:=\left[\omega\left(\frac{k z \bmod p^{m}}{p^{m}}\right)\right]_{\substack{z \in U_{p^{m}} \\ k \in \mathbb{Z}_{p}^{m}}},
$$


with $\omega(x)=\omega(1-x)$, can be ordered with respect to the divisors of $p^{m}$ as

$$
\boldsymbol{\Omega}_{p^{m}}^{\langle g\rangle}:=\left[\mathbf{1}_{p^{0}} \otimes \boldsymbol{B}_{p^{m}}^{\langle g\rangle}\left|\mathbf{1}_{p^{1}} \otimes \boldsymbol{B}_{p^{m-1}}^{\langle g\rangle}\right| \cdots\left|\mathbf{1}_{p^{m-1}} \otimes \boldsymbol{B}_{p^{1}}^{\langle g\rangle}\right| \mathbf{1}_{p^{m-1}(p-1)} \otimes \boldsymbol{B}_{p^{0}}^{\langle g\rangle}\right],
$$

where $g$ is a generator for $U_{p^{m}}$ and $\boldsymbol{B}_{p^{0}}^{\langle g\rangle}:=[\omega(0)]$. For $\ell \geq 1$, each matrix $\boldsymbol{B}_{p^{\ell}}^{\langle g\rangle}$ (associated with a divisor $p^{m-\ell}$ ) is circulant of the form

$$
\boldsymbol{B}_{p^{\ell}}^{\langle g\rangle}:=\left[\begin{array}{c|c}
\boldsymbol{M}_{p^{\ell}}^{\langle g\rangle} & \boldsymbol{M}_{p^{\ell}}^{\langle g\rangle} \\
\hline \boldsymbol{M}_{p^{\ell}}^{\langle g\rangle} & \boldsymbol{M}_{p^{\ell}}^{\langle g\rangle}
\end{array}\right],
$$

with circulant blocks

$$
\boldsymbol{M}_{p^{\ell}}^{\langle g\rangle}:=\left[\omega\left(\frac{k z \bmod p^{\ell}}{p^{\ell}}\right)\right]_{\substack{z \in\langle\langle g\rangle\rangle_{p^{\ell}} \\ k \in\left\langle\left\langle g^{-1}\right\rangle_{p^{\ell}}\right.}} .
$$

Here $\langle\langle g\rangle\rangle_{p^{\ell}}$ denotes the ordered set $\left[g^{i} \bmod p^{\ell}: 0 \leq i \leq \phi\left(p^{\ell}\right) / 2-1\right]$, i.e., half of the group $U_{p^{\ell}}$ in generator ordering.

THEOREM 4.3 (Block partitioning $\boldsymbol{\Omega}_{2^{m}}$ ). The matrix

$$
\boldsymbol{\Omega}_{2^{m}}:=\left[\omega\left(\frac{k z \bmod 2^{m}}{2^{m}}\right)\right]_{\substack{z \in U_{2^{m}} \\ k \in \mathbb{Z}_{2}^{m}}}
$$

with $\omega(x)=\omega(1-x)$, can be ordered with respect to the divisors of $2^{m}$ as

$$
\boldsymbol{\Omega}_{2^{m}}^{\langle 5\rangle}:=\left[\mathbf{1}_{2^{0}} \otimes \boldsymbol{B}_{2^{m}}^{\langle 5\rangle}\left|\mathbf{1}_{2^{1}} \otimes \boldsymbol{B}_{2^{m-1}}^{\langle 5\rangle}\right| \cdots\left|\mathbf{1}_{2^{m-1}} \otimes \boldsymbol{B}_{2^{1}}^{\langle 5\rangle}\right| \mathbf{1}_{2^{m-1}(2-1)} \otimes \boldsymbol{B}_{2^{0}}^{\langle 5\rangle}\right],
$$

where $\boldsymbol{B}_{2^{0}}^{\langle 5\rangle}:=[\omega(0)]$ and $\boldsymbol{B}_{2^{1}}^{\langle 5\rangle}:=[\omega(1 / 2)]$. For $\ell \geq 2$, each matrix $\boldsymbol{B}_{2^{\ell}}^{\langle 5\rangle}$ (associated with a divisor $\left.2^{m-\ell}\right)$ is circulant of the form

$$
\boldsymbol{B}_{2^{\ell}}^{\langle 5\rangle}:=\left[\begin{array}{c|c}
\boldsymbol{M}_{2^{\ell}}^{\langle 5\rangle} & \boldsymbol{M}_{2^{\ell}}^{\langle 5\rangle} \\
\hline \boldsymbol{M}_{2^{\ell}}^{\langle 5\rangle} & M_{2^{\ell}}^{j 5\rangle}
\end{array}\right],
$$

with circulant blocks

$$
\boldsymbol{M}_{2^{\ell}}^{\langle 5\rangle}:=\left[\omega\left(\frac{k z \bmod 2^{\ell}}{2^{\ell}}\right)\right]_{\substack{z \in\langle 5\rangle_{2} \ell \\ k \in\left\langle 5^{-1}\right\rangle_{2^{\ell}}}} .
$$

Here $\langle 5\rangle_{2^{\ell}}$ denotes the ordered set $\left[5^{i} \bmod 2^{\ell}: 0 \leq i \leq 2^{\ell-2}-1\right]$, i.e., half of the group $U_{2^{\ell}}$ in generator ordering.

We will illustrate this theorem with an example shortly. First we discuss the effect of the symmetry of $\omega$. Note that Theorem 4.3 rests upon the partition

$$
U_{2^{\ell}}=\langle 5\rangle_{2^{\ell}} \cup(-1)\langle 5\rangle_{2^{\ell}}, \quad \text { for } \ell \geq 2 .
$$

If we do not make use of the symmetry of $\omega$, formula (4.9) would actually look like

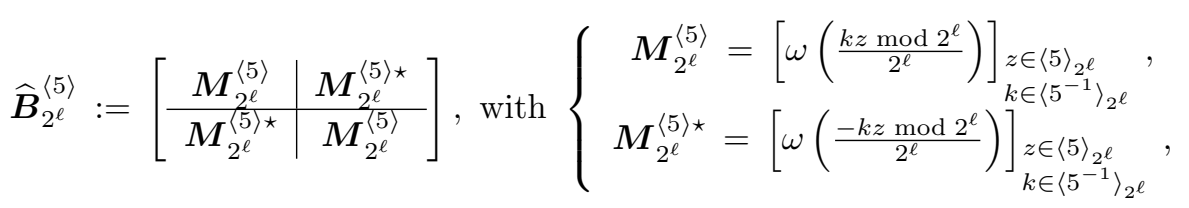


and the stacking of the matrices $\widehat{\boldsymbol{B}}_{2^{\ell}}^{\langle 5\rangle}$ would be

$$
\widehat{\boldsymbol{\Omega}}_{2^{m}}^{\langle 5\rangle}:=\left[\boldsymbol{R}_{2^{0}} \widehat{\boldsymbol{B}}_{2^{m}}^{\langle 5\rangle}|\cdots| \boldsymbol{R}_{2^{m-2}} \widehat{\boldsymbol{B}}_{2^{2}}^{\langle 5\rangle}\left|\mathbf{1}_{2^{m-1}} \otimes \boldsymbol{B}_{2^{1}}^{\langle 5\rangle}\right| \mathbf{1}_{2^{m-1}} \otimes \boldsymbol{B}_{2^{0}}^{\langle 5\rangle}\right],
$$

where $\boldsymbol{R}_{2^{\ell}}:=\boldsymbol{I}_{2} \otimes \mathbf{1}_{2^{\ell}} \otimes \boldsymbol{I}_{2^{m-\ell-2}}$, which stacks the top and bottom half of $\widehat{\boldsymbol{B}}_{2^{m-\ell}}^{\langle 5\rangle}$ separately. The symmetry of $\omega$ implies $\boldsymbol{M}_{2^{\ell}}^{\langle 5\rangle}=\boldsymbol{M}_{2^{\ell}}^{\langle 5\rangle}$ from which the theorem follows.

We consider now the example $n=2^{4}=16$, initially without using the symmetry of $\omega$. Following (4.10), the first half of $U_{16}$ is generated by 5

$$
\left[5^{i} \bmod 16: 0 \leq i \leq 3\right]=[1,5,9,13],
$$

where $i$ stays less than $\phi(n) / 2$ since this is only half of the group $U_{16}$. The second half is a 'copy' of this subgroup, but multiplied with $(-1) \equiv 15(\bmod 16)$

$$
\left[(-1) 5^{i} \bmod 16: 0 \leq i \leq 3\right]=[15,11,7,3] .
$$

With this row ordering we can associate a column ordering to get the circulant structure. This can be achieved by reversing the row ordering except for the first element. We obtain the first submatrix for the divisor $2^{0}=1$

$$
\widehat{\boldsymbol{B}}_{16}^{\langle 5\rangle}=\omega\left(\frac{1}{16}\left[\begin{array}{cccc|cccc}
1 & 13 & 9 & 5 & 15 & 3 & 7 & 11 \\
5 & 1 & 13 & 9 & 11 & 15 & 3 & 7 \\
9 & 5 & 1 & 13 & 7 & 11 & 15 & 3 \\
13 & 9 & 5 & 1 & 3 & 7 & 11 & 15 \\
\hline 15 & 3 & 7 & 11 & 1 & 13 & 9 & 5 \\
11 & 15 & 3 & 7 & 5 & 1 & 13 & 9 \\
7 & 11 & 15 & 3 & 9 & 5 & 1 & 13 \\
3 & 7 & 11 & 15 & 13 & 9 & 5 & 1
\end{array}\right]\right)
$$

The submatrices for the other divisors can be constructed similarly, and we get

$$
\widehat{\boldsymbol{B}}_{8}^{\langle 5\rangle}=\omega\left(\frac{1}{8}\left[\begin{array}{ll|ll}
1 & 5 & 7 & 3 \\
5 & 1 & 3 & 7 \\
\hline 7 & 3 & 1 & 5 \\
3 & 7 & 5 & 1
\end{array}\right]\right), \quad \widehat{\boldsymbol{B}}_{4}^{\langle 5\rangle}=\omega\left(\frac{1}{4} \frac{1}{3} \mid \frac{3}{3}\right), \quad \boldsymbol{B}_{2}^{\langle 5\rangle}=\omega\left(\begin{array}{llll}
\frac{1}{2} & 1
\end{array}\right), \quad \boldsymbol{B}_{1}^{\langle 5\rangle}=\omega\left(\begin{array}{ll} 
& 0
\end{array}\right) .
$$

Note that all these matrices (except for the last two trivial ones) have a blockcirculant-with-circulant-blocks structure, which is consistent with the two partitions in their multiplicative groups (see (4.10)). Using (4.11), the full matrix looks like this (where italic font means redundancy)

$$
\begin{aligned}
& \widehat{\mathbf{\Omega}}_{16}^{\langle 5\rangle}=
\end{aligned}
$$

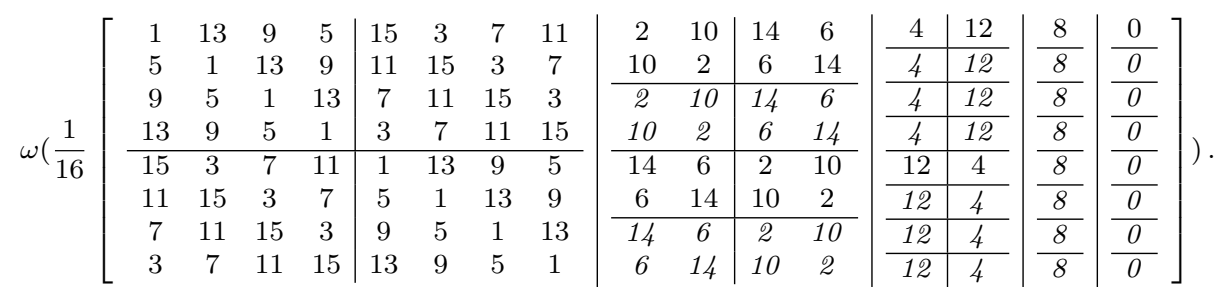

We now apply the symmetry of $\omega$ by mapping each $k \in \mathbb{Z}_{n}$ to $\min (n-k, k)$ and we get the matrix

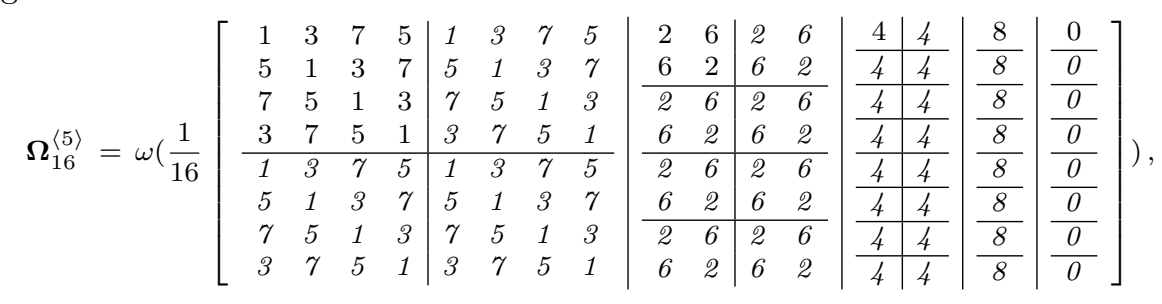


as promised in Theorem 4.3. We can then represent this matrix in a reduced form

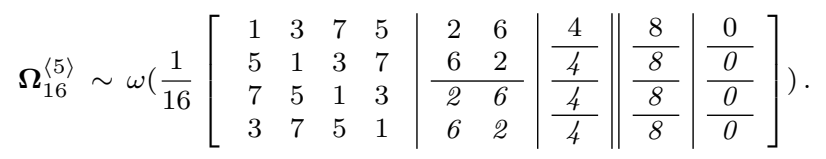

If we write out the reordering from Theorems 4.2 and 4.3 explicitly by means of permutations $\Pi_{g}^{\top}$ and $\Pi_{g^{-1}}$ on the rows and columns induced by $g$, we obtain

$$
\boldsymbol{y}=\boldsymbol{\Omega}_{p^{m}} \boldsymbol{x} \quad \Leftrightarrow \quad \Pi_{g}^{\top} \boldsymbol{y}=\underbrace{\Pi_{g}^{\top} \boldsymbol{\Omega}_{p^{m}} \Pi_{g^{-1}}}_{\boldsymbol{\Omega}_{p^{m}}^{\langle g\rangle}} \Pi_{g^{-1}}^{\top} \boldsymbol{x}=\boldsymbol{\Omega}_{p^{m}}^{\langle g\rangle} \Pi_{g^{-1}}^{\top} \boldsymbol{x} .
$$

Note that the permutations do not complicate matters, since the starting vectors are all ones and we know that the vectors

$$
\begin{array}{lll}
\boldsymbol{p}_{d}^{\left\langle g^{-1}\right\rangle}:=\Pi_{g^{-1}}^{\top} \boldsymbol{p}_{d}=\left(\mathbf{1}_{n}+\gamma_{d} \boldsymbol{\Omega}_{p^{m}}^{\langle g\rangle}\left(z_{d}\right)\right) * \boldsymbol{p}_{d-1}^{\left\langle g^{-1}\right\rangle} & \text { (product) } \\
\boldsymbol{p}_{d, \ell}^{\left\langle g^{-1}\right\rangle}:=\Pi_{g^{-1}}^{\top} \boldsymbol{p}_{d, \ell}=\boldsymbol{p}_{d-1, \ell}^{\left\langle g^{-1}\right\rangle}+\boldsymbol{\Omega}_{p^{m}}^{\langle g\rangle}\left(z_{d}\right) * \boldsymbol{p}_{d-1, \ell-1}^{\left\langle g^{-1}\right\rangle} & \text { (order-dependent) }
\end{array}
$$

have the same symmetry structure as the rows of the matrix $\boldsymbol{\Omega}_{p^{m}}^{\langle g\rangle}$, which means that we can keep a reduced version in memory and never have to refer to the unpermuted version. Also, from the resulting vector $\boldsymbol{E}_{d}^{2}$ we only need the index of the minimal entry, from which we obtain $z_{d}$ via the sequence $\langle g\rangle_{p^{m}}$.

Combining this with Lemma 4.1, we observe that we only need the fast Fourier transforms of the first column of the smaller circulant matrices in the reduced matrix. This requires $\mathcal{O}(n)$ memory. A matrix-vector product with a vector of length $n \times 1$ involves $m+1$ (the number of divisors) smaller matrix-vector products with circulant matrices, with a total cost of

$$
\sum_{\ell=0}^{m} \phi\left(p^{m-\ell}\right) \log \left(\phi\left(p^{m-\ell}\right)\right)=\mathcal{O}(n \log (n)) .
$$

We can calculate all these circulant matrix-vector products in turn, starting from the smallest matrix up to the largest matrix, whilst accumulating the results. In each step the previous result vector is replicated $p-1$ times before the next result vector is added. The summing alone has a memory complexity of $\mathcal{O}(n)$ and a time complexity of $\mathcal{O}(n)$, which is negligible against the $\mathcal{O}(n \log (n))$ cost of the matrix-vector products. Again we specify this as a specialized version of the general case in [14, Theorem 5]. (There is no need for a special summing order as is the case for general $n$ in [14].)

THEOREM 4.4 (Fast matrix-vector product $\boldsymbol{\Omega}_{p^{m}} \boldsymbol{p}$ ). The matrix-vector product $\boldsymbol{\Omega}_{p^{m}} \boldsymbol{p}$ for the vectors $\boldsymbol{p}$ defined in (4.7) and (4.8) can be done in the permuted space in $\mathcal{O}(n \log (n))$ time with $\mathcal{O}(n)$ memory by doing each of the matrix-vector products with the matrices $\boldsymbol{B}_{p^{\ell}}^{\langle g\rangle}$ in a fast way.

4.3. Exploiting the embedding structure. In Theorems 4.2 and 4.3 we used implicitly the property that a generator $g$ for the group $U_{p^{m}}$ also generates $U_{p^{\ell}}$ for all $\ell \leq m$, with the obvious adjustment for $p=2$ where 5 generates $U_{2^{\ell}}$ in two partitions for all $\ell \leq m$. This actually follows from the fact (see e.g. [1, §24.3.4]) that if we have a generator $r$ for $U_{p}$, then a generator $g$ for $U_{p^{\ell}}, \ell \geq 2$, can be found by taking

$$
g= \begin{cases}r+p, & \text { if } r^{p-1} \equiv 1\left(\bmod p^{2}\right) \\ r, & \text { otherwise }\end{cases}
$$


It can be checked that if one searches for generators in increasing numerical order starting from 2 , then the first $p$ for which the smallest generator of $U_{p}$ does not generate $U_{p^{2}}$ is as high as 40487 (and the first $p$ for which one of its generators does not generate $U_{p^{2}}$ is $p=29$ ). In other words, as long as $p$ is smaller than 40487, the smallest generator of $U_{p}$ also generates $U_{p^{e}}$ for all $\ell \geq 1$. Furthermore, the most logical choice in practice is $p=2$ for which we can always pick 5 as a pseudo-generator. This gives us a way to extend a matrix for $p^{m}$ points to a matrix for $p^{m+1}$ points by simply adding one block in front.

We now show the extension to $\boldsymbol{\Omega}_{32}^{\langle 5\rangle}$ starting from $\boldsymbol{\Omega}_{16}^{\langle 5\rangle}$ :

$$
\boldsymbol{\Omega}_{32}^{\langle 5\rangle}=\left[\begin{array}{l|l}
\boldsymbol{B}_{32}^{\langle 5\rangle} & \boldsymbol{\Omega}_{16}^{\langle 5\rangle} \\
\hline \boldsymbol{\Omega}_{16}^{\langle 5\rangle}
\end{array}\right],
$$

where we have recursively

$$
\boldsymbol{\Omega}_{16}^{\langle 5\rangle}=\left[\begin{array}{l|l}
\boldsymbol{B}_{16}^{\langle 5\rangle} & \boldsymbol{\Omega}_{8}^{\langle 5\rangle} \\
\boldsymbol{\Omega}_{8}^{\langle 5\rangle}
\end{array}\right], \boldsymbol{\Omega}_{8}^{\langle 5\rangle}=\left[\begin{array}{l|l}
\boldsymbol{B}_{8}^{\langle 5\rangle} & \boldsymbol{\Omega}_{4}^{\langle 5\rangle} \\
\boldsymbol{\Omega}_{4}^{\langle 5\rangle}
\end{array}\right], \boldsymbol{\Omega}_{4}^{\langle 5\rangle}=\left[\begin{array}{l|l}
\boldsymbol{B}_{4}^{\langle 5\rangle} & \boldsymbol{\Omega}_{2}^{\langle 5\rangle} \\
\boldsymbol{\Omega}_{2}^{\langle 5\rangle}
\end{array}\right],
$$

with $\boldsymbol{\Omega}_{2}^{\langle 5\rangle}=\left[\boldsymbol{B}_{2}^{\langle 5\rangle} \mid \boldsymbol{B}_{1}^{\langle 5\rangle}\right]$. In reduced form (and a common scaling of 1/32) we have

$$
\begin{aligned}
& \boldsymbol{\Omega}_{32}^{\langle 5\rangle} \sim
\end{aligned}
$$

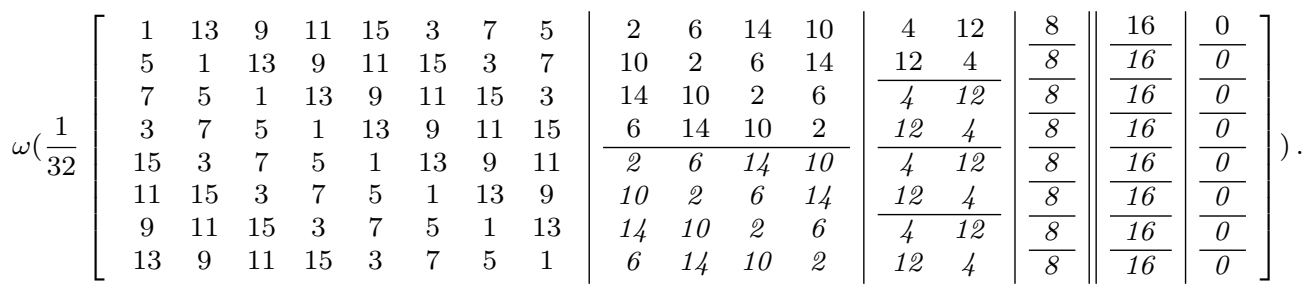

In $\S 4.2$ we explained how to sum up the result vectors of a matrix-vector multiplication with the matrix $\boldsymbol{\Omega}_{p^{m}}$ in $\mathcal{O}(n \log (n))$ time. In fact, since $n$ is of the form $p^{m}$, any method used to sum up the intermediate result vectors would have been sufficient to get at the $\mathcal{O}(n \log (n))$ time complexity (see [14]). By accumulating the result vectors from the smallest matrix upwards, we obtain intermediate result vectors which are precisely the matrix-vector products with the matrices $\boldsymbol{\Omega}_{p^{e}}$ for $\ell$ going from 0 up to $m-1$. Thus in an attempt to compute $e_{p^{m}, d}$, we get along the way the values of $e_{p^{\ell}, d}$ for $\ell$ from 0 to $m-1$ at no extra cost.

The availability of all these intermediate results is a nice side effect since then the cost for calculating $X_{m_{1}, m_{2}, d}$, which involves $e_{p^{\ell}, d}$ for all $\ell$ from $m_{1}$ to $m_{2}$, is no worse than the cost for calculating just $e_{p^{m_{2}, d}}$. This brings us to the main result of this paper.

TheOREm 4.5 (Fast construction of embedded lattice rules). Construction of embedded lattice rules up to $n=p^{m_{2}}$ points, with $p$ prime, using Algorithm 2 can be done in $\mathcal{O}(d n \log (n))$ time, with $\mathcal{O}(n)$ memory for product weights and $\mathcal{O}(q n)$ memory for order-dependent weights of order $q$. The total construction cost including the precalculations of Algorithm 1 is therefore $\mathcal{O}\left(\operatorname{dn}(\log (n))^{2}\right)$.

\section{Using embedded lattice rules.}

5.1. Ordering of lattice points. The main advantage of having a sequence of embedded lattice rules $L_{0} \subset L_{1} \subset L_{2} \subset \cdots$ is that we can increase the number of points without having to throw away already computed function values. When the 
base is $b$ (not necessary prime anymore), we increase the number of points by a factor of $b$ each step along the sequence. For $\ell \geq 1$, let $\Delta L_{\ell}:=L_{\ell} \backslash L_{\ell-1}$ denote the set of additional points from the point set $L_{\ell-1}$ to $L_{\ell}$. We recall from Property 4 that the points in $L_{\ell-1}$ are generated by the indices $k$ which are multiples of $b$ in $\mathbb{Z}_{b^{\ell}}$. Thus we can write

$$
L_{m}=L_{0} \cup\left(\bigcup_{\ell=1}^{m} \Delta L_{\ell}\right)
$$

with $L_{0}=\{\mathbf{0}\}$ and

$$
\Delta L_{\ell}=\left\{\left\{\frac{k \boldsymbol{z}}{b^{\ell}}\right\}: k \in\left(\mathbb{Z}_{b^{\ell}} \backslash b \mathbb{Z}_{b^{\ell-1}}\right)\right\} .
$$

Letting $m \rightarrow \infty$ in (5.1) leads formally to an extensible lattice sequence $L_{\infty}$ with an infinite number of points. In this paper we consider lattice point sets where the number of points is finite, that is, the union in (5.1) only goes to a predefined upper limit $m_{2}$. But, due to the fast construction, this limit can be taken rather large.

Note that the set of additional points $\Delta L_{\ell}$ from the point set $L_{\ell-1}$ to $L_{\ell}$ actually comprises of $b-1$ shifted versions of $L_{\ell-1}$. We put this forward as another property of embedded lattice rules (see also [7, Theorem 2.3]).

Property 5. The lattice point set $L_{m}$ can be considered as the union of $b$ shifted versions (dependent on $\boldsymbol{z}$ ) of the lattice point set $L_{m-1}$, as well as the union of $b^{2}$ shifted versions of $L_{m-2}$, and so on. Trivially, $L_{m}$ is the union of $b^{m}$ shifted versions of the initial point $\mathbf{0}$.

Explicitly, we have

$$
L_{m}=\bigcup_{k_{0}=0}^{b-1} \cdots \bigcup_{k_{\ell}=0}^{b-1}\left(L_{m-\ell-1}+\frac{k_{\ell} \boldsymbol{z}}{b^{m-\ell}}+\cdots+\frac{k_{0} \boldsymbol{z}}{b^{m}}\right) \quad \text { for all } \ell=0, \ldots, m-1,
$$

where the addition is interpreted as in the definition $P_{n}+\boldsymbol{\Delta}:=\left\{\{\boldsymbol{t}+\boldsymbol{\Delta}\}: \boldsymbol{t} \in P_{n}\right\}$ (the inner braces indicate taking the fractional part).

With the generating vector constructed by Algorithm 2 for $n$ ranging from $b^{m_{1}}$ to $b^{m_{2}}$, we can start with an approximation of the integral using the point set $L_{m_{1}}$, and then gradually add blocks of additional points $\Delta L_{m_{1}+1}, \Delta L_{m_{1}+2}, \ldots, \Delta L_{m_{2}}$ until we are satisfied with the error (provided that $m_{2}$ is large enough). This usage of the sequence of embedded lattice rules is justified by the knowledge that every lattice point set $L_{m}$ is good for $m_{1} \leq m \leq m_{2}$.

A more interesting usage would be to apply the lattice rule one point at a time, i.e., as a low-discrepancy sequence. Since we may end up with an incomplete lattice point set, there is no guarantee that these additional points will be well distributed over the unit cube, and we could even end up with these points all falling in one half of the cube. In fact, we are sure to get a bad ordering of the points if we generate the points from each block $\Delta L_{\ell}$ according to the natural order of the $k$ indices in (5.2). The solution to this inappropriate ordering of the points is given by replacing $k / b^{\ell}$ in (5.2) with the radical inverse function, possibly in combination with Gray code ordering. These schemes provide a better ordering of the numbers $k / b^{\ell}$ in the unit interval, and this property is preserved under multiplication by $\boldsymbol{z}$ (and then taking the fractional part) so that the ordering of every one-dimensional projection of the lattice points is good. Both schemes are well-known tools borrowed from the vast literature on digital sequences, see for example [12]. 
The radical inverse function takes an integer in base $b$ and mirrors it around the radix point. For an integer $k=\sum_{\ell=0}^{m-1} k_{\ell} b^{\ell}=\left(k_{m-1} \ldots k_{0}\right)_{b}$ the radical inverse is given as $\phi_{b}(k)=\sum_{\ell=0}^{m-1} k_{\ell} b^{-(\ell+1)}=\left(0 . k_{0} k_{1} \ldots k_{m-1}\right)_{b}$. The Gray code variant uses this same transformation, but starts with a Gray code ordering in base $b$ of the $k$ indices (i.e., successive indices only differ in one $b$-ary digit). The latter is computationally more efficient since then the next index can be obtained from the previous one by a simple digit change and the actual radical inversion can be avoided.

We summarize the usage of these schemes as a property of embedded lattice rules. Note that they preserve the embedding property $L_{0} \subset L_{1} \subset \cdots$ of the lattice point sets, while providing a better ordering of the points within each block of additional points $\Delta L_{\ell}$. More discussions can be found in [7].

Property 6. The lattice point set $L_{m}$ can be used as a low-discrepancy sequence if the points are generated in the order

$$
\boldsymbol{t}_{k}=\left\{\varphi_{b}(k) \boldsymbol{z}\right\} \quad \text { for } \quad k=0,1, \ldots, b^{m}-1,
$$

where $\varphi_{b}(k)$ denotes either the radical inverse $\phi_{b}(k)$ of $k$ in base $b$, or the corresponding Gray code variant.

We end this subsection with a simple illustration for the most practical case of $b=2$ :

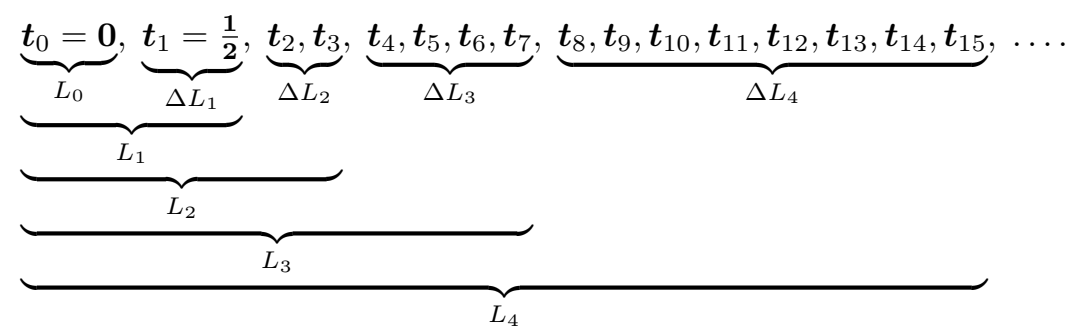

The number of points doubles as we move along the sequence, and the set of additional points $\Delta L_{\ell}$ is generated by $\left\{k \boldsymbol{z} / 2^{\ell}\right\}$ with $k$ running through all the odd numbers up to $2^{\ell}-1$. In Table 5.1 we show how the points are ordered under radical inverse and the Gray code variant.

5.2. Error estimation. There is a comprehensive discussion on error estimation in [7] including error estimation based on the embedding structure of the lattice rules. Here we only focus on the simplest form of error estimation using random shifts.

In practice, once we have found a good generating vector $\boldsymbol{z}$, we generate a number of independent random shifts $\boldsymbol{\Delta}_{1}, \boldsymbol{\Delta}_{2}, \ldots, \boldsymbol{\Delta}_{w}$ and compute the approximations $Q_{1}, Q_{2}, \ldots, Q_{w}$, where $Q_{i}$ is the shifted lattice rule approximation to the integral $I_{d}(f)$ based on the generating vector $\boldsymbol{z}$ and the shift $\boldsymbol{\Delta}_{i}$. Then we take the average

$$
\bar{Q}:=\frac{1}{w}\left(Q_{1}+Q_{2}+\cdots+Q_{w}\right)
$$

as our final approximation to $I_{d}(f)$. An unbiased estimate for the standard error of our approximation is given by

$$
\hat{\sigma}:=\sqrt{\frac{1}{w(w-1)} \sum_{i=1}^{w}\left(Q_{i}-\bar{Q}\right)^{2}} .
$$

Using the Chebyshev inequality we can obtain a $\left(100-100 / v^{2}\right) \%$ confidence interval $\bar{Q} \pm v \hat{\sigma}($ with $v>1$ ). 
TABLE 5.1

Ordering of lattice points

\begin{tabular}{|c|c|c|c|c|}
\hline & $k$ & natural order & radical inverse & Gray code variant \\
\hline$L_{0}$ & 0 & 0 & 0 & 0 \\
\hline$\Delta L_{1}$ & 1 & $1 / 2=0.5$ & $(0.1)_{2}=0.5$ & $(0.1)_{2}=0.5$ \\
\hline$\Delta L_{2}$ & 2 & $1 / 4=0.25$ & $(0.01)_{2}=0.25$ & $(0.11)_{2}=0.75$ \\
& 3 & $3 / 4=0.75$ & $(0.11)_{2}=0.75$ & $(0.01)_{2}=0.25$ \\
\hline$\Delta L_{3}$ & 4 & $1 / 8=0.125$ & $(0.001)_{2}=0.125$ & $(0.011)_{2}=0.375$ \\
& 5 & $3 / 8=0.375$ & $(0.101)_{2}=0.625$ & $(0.111)_{2}=0.875$ \\
& 6 & $5 / 8=0.625$ & $(0.011)_{2}=0.375$ & $(0.101)_{2}=0.625$ \\
& 7 & $7 / 8=0.875$ & $(0.111)_{2}=0.875$ & $(0.001)_{2}=0.125$ \\
\hline$\Delta L_{4}$ & 8 & $1 / 16=0.0625$ & $(0.0001)_{2}=0.0625$ & $(0.0011)_{2}=0.1875$ \\
& 9 & $3 / 16=0.1875$ & $(0.1001)_{2}=0.5625$ & $(0.1011)_{2}=0.6875$ \\
& 10 & $5 / 16=0.3125$ & $(0.0101)_{2}=0.3125$ & $(0.1111)_{2}=0.9375$ \\
& 11 & $7 / 16=0.4375$ & $(0.1101)_{2}=0.8125$ & $(0.0111)_{2}=0.4375$ \\
& 12 & $9 / 16=0.5625$ & $(0.0011)_{2}=0.1875$ & $(0.0101)_{2}=0.3125$ \\
& 13 & $11 / 16=0.6875$ & $(0.1011)_{2}=0.6875$ & $(0.1101)_{2}=0.8125$ \\
& 14 & $13 / 16=0.8125$ & $(0.0111)_{2}=0.4375$ & $(0.1001)_{2}=0.5625$ \\
& 15 & $15 / 16=0.9375$ & $(0.1111)_{2}=0.9375$ & $(0.0001)_{2}=0.0625$ \\
\hline$\vdots$ & & \multicolumn{3}{|l}{} \\
\hline
\end{tabular}

\section{Numerical experiments.}

6.1. Results from CBC construction. We construct good generating vectors of embedded lattice rule up to 360 dimensions using Algorithm 2 for $n$ ranging from $2^{10}$ to $2^{20} \approx 1$ million (i.e. $b=2, m_{1}=10$, and $m_{2}=20$ ). Our search criterion is the quantity $X_{m_{1}, m_{2}, d}(\boldsymbol{z})$ defined in (1.3), i.e.,

$$
X_{10,20, d}(\boldsymbol{z})=\max _{10 \leq m \leq 20} \frac{e_{2^{m}, d}(\boldsymbol{z})}{e_{2^{m}, d}\left(\boldsymbol{z}^{(m)}\right)},
$$

where $\boldsymbol{z}^{(m)}$ is the generating vector constructed using Algorithm 1 for a $2^{m}$-point rule (which we refer to as a good "fixed" rule). Let $\boldsymbol{z}^{\star}$ denote the vector resulting from Algorithm 2. Then the quantity $X_{10,20, d}\left(\boldsymbol{z}^{\star}\right)$ tells us how far off the vector $\boldsymbol{z}^{\star}$ is compared with the vectors $\boldsymbol{z}^{(m)}$ in terms of the maximum (over $m$ ) ratio of the worst-case errors.

We consider weighted Sobolev spaces (see Example 2.2) with both product weights and order-dependent weights. In particular, we consider equal product weights, decaying product weights, order- 2 weights and order- 3 weights. Our choices of weights together with the maxima of $X_{10,20, d}$ over all $1 \leq d \leq 360$ are given in Table 6.1.

TABLE 6.1

Maximum of $X_{10,20, d}\left(\boldsymbol{z}^{\star}\right)$ for various weights

\begin{tabular}{|l|c|}
\hline Weights & $\max _{d} X_{10,20, d}\left(\boldsymbol{z}^{\star}\right)$ \\
\hline product 1 & 1.53 \\
product 0.5 & 1.48 \\
product 0.3 & 1.47 \\
product 0.1 & 1.41 \\
product 0.05 & 1.37 \\
product 0.01 & 1.30 \\
product $0.9^{j}$ & 1.50 \\
product $0.75^{j}$ & 1.42 \\
product $0.5^{j}$ & 1.30 \\
\hline
\end{tabular}

\begin{tabular}{|c|c|}
\hline Weights & $\max _{d} X_{10,20, d}\left(\boldsymbol{z}^{\star}\right)$ \\
\hline product $1 / j$ & 1.41 \\
\hline product $1 / j^{2}$ & 1.31 \\
\hline order-2 & 1.43 \\
\hline order-3 1 & 1.42 \\
\hline order-3 $\quad 0.5$ & 1.55 \\
\hline order-3 0.3 & 1.45 \\
\hline order-3 0.1 & 1.47 \\
\hline order-3 0.05 & 1.45 \\
\hline order-3 0.01 & 1.43 \\
\hline
\end{tabular}


The product-type weights in the table are self-explanatory: "product 0.5 " means $\gamma_{j}=0.5$ and "product $1 / j$ " means $\gamma_{j}=1 / j$. We shall explain briefly our choices of order- 2 and order- 3 weights. Firstly, since all one-dimensional projections of our lattice rules are left-rectangle rules with all points distinct, the weight $\Gamma_{1}$ in an orderdependent weight setting has no essential effect on the choice of the rule. We thus set $\Gamma_{1}=1$. Secondly, if we decide to construct a rule based on order-2 weights (i.e. if $\Gamma_{\ell}=0$ for $\ell>2$ ), then the rule is unique regardless of how we choose $\Gamma_{2}$, since in this case $\Gamma_{2}$ only rescales the worst-case error. Therefore without lost of generality we set $\Gamma_{2}=1$. In other words, such a rule is universally applicable for all order-2 problems. For order-3 weights, the rule depends only on the ratio $\Gamma_{3} / \Gamma_{2}$. Hence we use $\Gamma_{3}$ to specify the importance of order-3 interactions compared with order-2 interactions. In our table "order-3 0.1 " corresponds to the choice $\Gamma_{1}=\Gamma_{2}=1$ and $\Gamma_{3}=0.1$. Note that having equal product weights $\gamma_{j}=r$ is equivalent to having order-dependent weights with $\Gamma_{\ell}=r^{\ell}$. The generating vector for the order-2 rule is given in the Appendix. From Table 6.1, the worst-case errors for the order-2 rule in the Appendix are within a factor of 1.43 of the worst-case errors obtained with the results from the original $\mathrm{CBC}$ construction.

Starting from $X_{10,20,1}\left(\boldsymbol{z}^{\star}\right)=1$, the empirical observation is that in most cases the numbers $X_{10,20, d}\left(\boldsymbol{z}^{\star}\right)$ increase initially as $d$ increases, then they decrease from some dimension onward. We see from Table 6.1 that $X_{10,20, d}\left(\boldsymbol{z}^{\star}\right)$ is substantially less than 1.6 for all our choices of weights. Thus we conclude that, in all our experiments, the embedded lattice rules are at most 1.6 times worse than the near-optimal rules constructed from Algorithm 1 for specific values of $n$. Put differently, we have rules with the optimal rate of convergence, but with a constant which is a factor of at most 1.6 times larger.

Enlarging the range of $m$ reduces the overall quality. Fig. 6.1 shows the profile of $X_{10, m_{2}, d}\left(\boldsymbol{z}^{\star}\right)$ with increasing $d$ for order-2 rules with $m_{2}=15,20$, and 25 . It is understandable that by allowing the range of $n$ to grow, the quality of the point set is sacrificed. But we observe that the quality remains very good for all feasible values of $m_{2}$.

Using initial segments of a good embedded lattice rule works as expected. The criterion $X_{10,20, d}\left(\boldsymbol{z}^{\star}\right)$ ensures that the resulting vector $\boldsymbol{z}^{*}$ is good for a $2^{m}$-point rule for all values of $m$ between 10 and 20. In other words, every point set in the embedded sequence $L_{10} \subset L_{11} \subset \cdots \subset L_{20}$ is good. (We refer to the smaller lattice point sets $L_{\ell}$ with $\ell<20$ as the "initial segments" of the point set $L_{20}$.) In Fig. 6.2 we plot the worst-case error ratios

$$
\frac{e_{2^{m}, d}\left(\boldsymbol{z}^{\star}\right)}{e_{2^{m}, d}\left(\boldsymbol{z}^{(m)}\right)}
$$

for the case of the order- 2 rule in the Appendix and every $m=10, \ldots, 20$.

As expected, the maximum of all these graphs corresponds to the graph for $X_{10,20, d}\left(\boldsymbol{z}^{\star}\right)$ in Fig. 6.1. For most values of $m$ we do better than $X_{10,20, d}\left(\boldsymbol{z}^{\star}\right)$ promises, but we note that all the individual curves actually stay close to $X_{10,20, d}\left(\boldsymbol{z}^{\star}\right)$. We also see that these curves cross each other in an erratic manner, which is important, otherwise we could redefine the error measure to make use of some assumed ordering. That said, in general it can be observed that the curves for higher $m$ tend to be closer to the top.

For high dimensions we see that the curves for $m=10$ and $m=12$ depart from the general trend. This might be explained by observing that the differences 


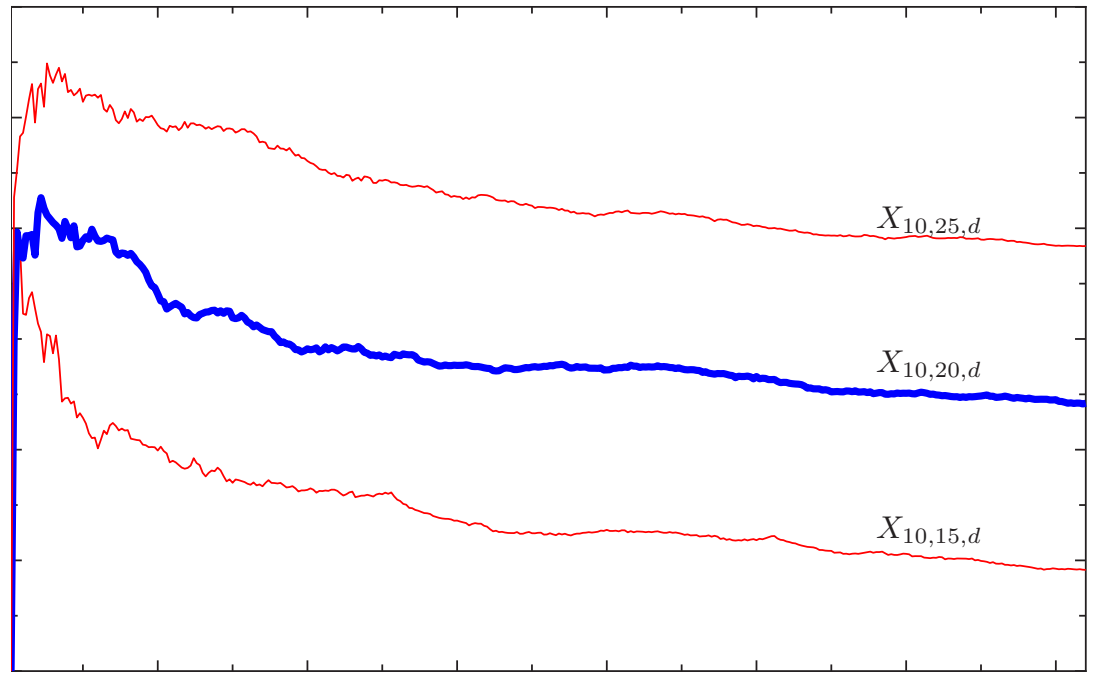

FIG. 6.1. Profile of $X_{10, m_{2}, d}\left(\boldsymbol{z}^{\star}\right)$ for order-2 rules with $m_{2}=15,20$, and 25 as $d$ increases

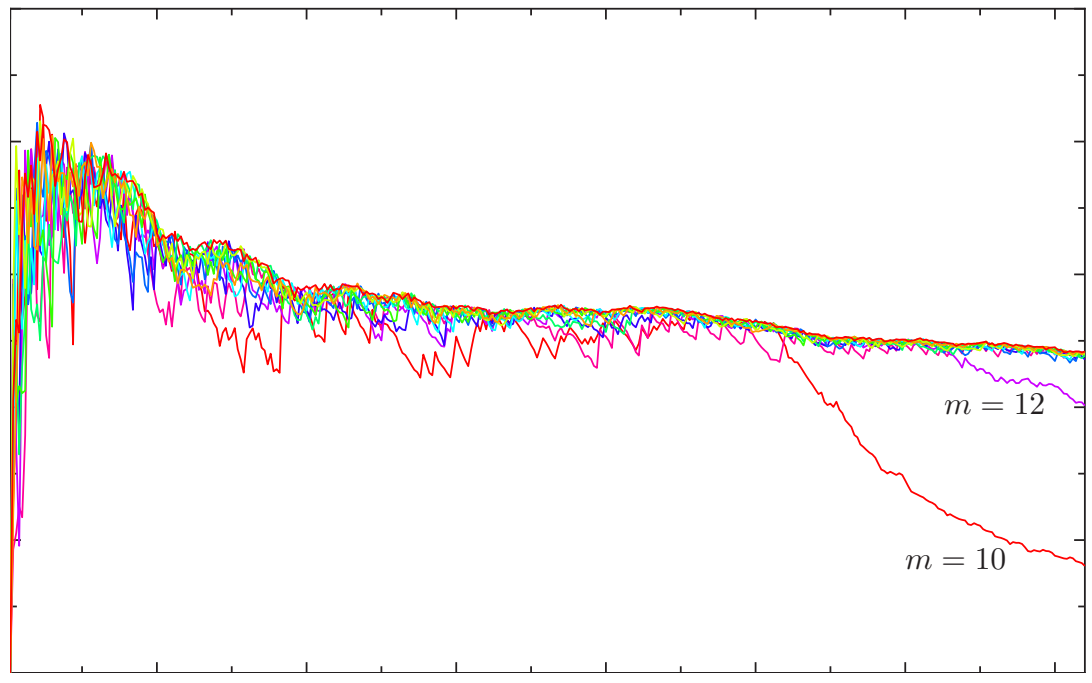

FIG. 6.2. Initial segments of a good embedded lattice rule

between the worst-case errors of different choices for a new component decrease as the dimension increases. In other words, the different choices of generating vectors are equally bad when there are very few points in very high dimensions.

Using initial segments of a good fixed lattice rule does not work. We could wonder if it would be satisfactory to just use the initial segments from a good 


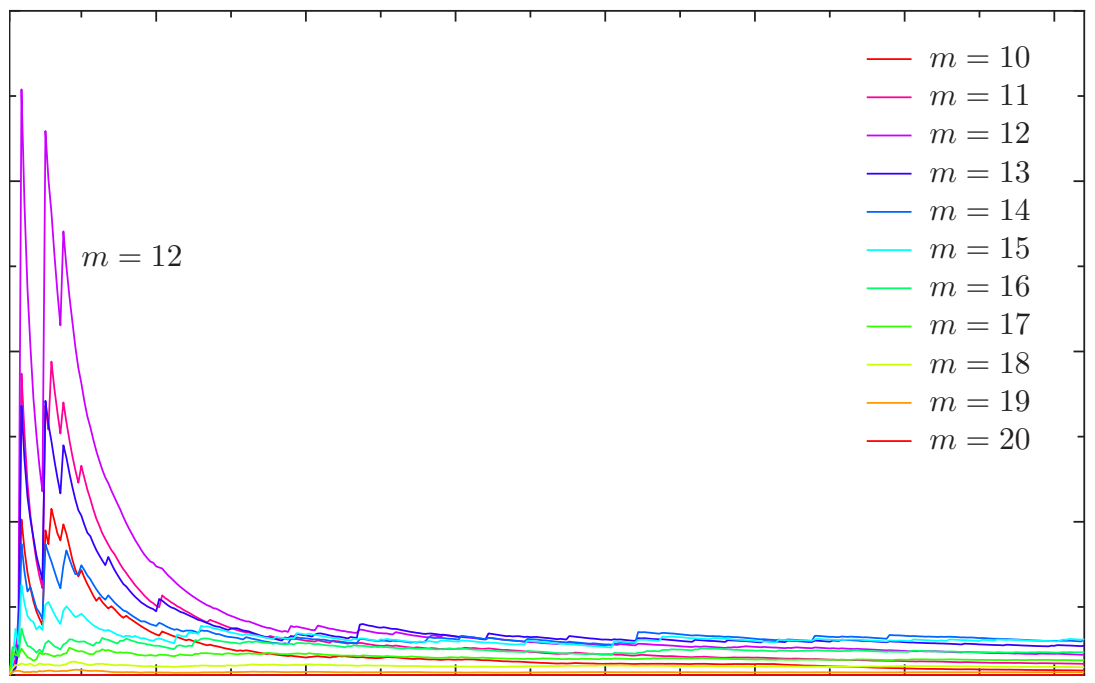

FIG. 6.3. Initial segments of a good fixed lattice rule

$2^{20}$-point lattice rule with generating vector $\boldsymbol{z}^{(20)}$ constructed by Algorithm 1. (As before, by initial segments we mean the smaller lattice point sets $L_{\ell}$ embedded in $L_{20}$ with $\ell<20$.) If these initial segments would be any good, then there would be little point to use Algorithm 2. In Fig. 6.3 we plot the worst-case error ratios

$$
\frac{e_{2^{m}, d}\left(\boldsymbol{z}^{(20)}\right)}{e_{2^{m}, d}\left(\boldsymbol{z}^{(m)}\right)}
$$

for every $m=10, \ldots, 20$.

It is obvious from the figure that using a large good fixed lattice rule for a maximum number of points $n=2^{m_{2}}$ tells you nothing about the goodness of the initial segments of this lattice rule. While $X_{10,20, d}\left(\boldsymbol{z}^{\star}\right)$ is bounded by 1.45 for the good embedded lattice rule, for the fixed lattice rule, the corresponding ratio is 35 times worse than the optimal fixed lattice rule for $m=12$. Once again there is no direct ordering in these curves, but it is obvious that the curves corresponding to higher $m$ are lower on the graph. For $m=20$ we have a horizontal line at 1 , and the line just above this constant line is the one for $m=19$. It is not surprising that using half of the points of a good fixed lattice rule would also give a reasonable rule. The maximum of all these curves is clearly very bad. We can draw a very clear conclusion from this graph: the embedded lattice rule constructed by Algorithm 2 works very well in comparison with a more simple-minded strategy.

6.2. Pricing an Asian option. We test the performance of the embedded lattice rules constructed in this paper for calculating the pricing of an Asian call option. This problem is extensively documented, both in the financial literature and in the QMC literature $[9,11,2]$. For an Asian option (also called average option) the payoff is determined by the average value of the underlier (i.e. a stock price) during its life time. We will consider a (European style) Asian option with arithmetic average of 
$d$ equally spaced stock prices $S_{j}$ at times $t_{j}=j T / d$, with expiry time $T$. The payoff for such a call option can be written as

$$
C(T)=\max \left(\frac{1}{d} \sum_{j=1}^{d} S_{j}-K, 0\right)=\left(\frac{1}{d} \sum_{j=1}^{d} S_{j}-K\right)^{+},
$$

where $K$ is the strike price at the expiry time $T=t_{d}$. We assume the risk-neutral measure of the Black-Scholes model for the path of the stock prices

$$
S_{j}=S_{0} \exp \left(\left(r-\sigma^{2} / 2\right) t_{j}+\sigma w_{j}\right)
$$

where $S_{0}$ is the initial stock price, $r$ is the risk-free interest rate, $\sigma$ the volatility of the stock price and the $w_{j}$ are samples of a Brownian motion a times $t_{j}$, i.e. $w_{j}$ is normally distributed with variance $t_{j}$, or $w_{j} \sim \mathcal{N}\left(0, t_{j}\right)$. This sampled Brownian motion has mean 0 and covariance matrix $\boldsymbol{\Sigma}=\left[\min \left(t_{i}, t_{j}\right)\right]_{i, j=1, \ldots, d}$.

The value of the Asian option at time 0 can then be written as the actualized expected payoff at time $T$,

$$
\begin{aligned}
C(0) & =\mathbb{E}(\exp (-r T) C(T)) \\
& =\exp (-r T) \int_{\mathbb{R}^{d}}\left(\frac{1}{d} \sum_{j=1}^{d} S_{0} \exp \left(\left(r-\sigma^{2} / 2\right) t_{j}+\sigma w_{j}\right)-K\right)^{+} \mathcal{N}(\boldsymbol{w} ; \mathbf{0}, \boldsymbol{\Sigma}) \mathrm{d} \boldsymbol{w} .
\end{aligned}
$$

We transform this integral to $[0,1]^{d}$ by using the inverse of the cumulative normal distribution function, $\Phi^{-1}$, and deal with the correlation in our sample points by using a factorization of the covariance matrix, $\boldsymbol{\Sigma}=\boldsymbol{A}^{\top} \boldsymbol{A}$. Then our lattice rule approximation to $C(0)$ is given by

$$
\widetilde{C}(0)=\frac{\exp (-r T)}{n} \sum_{k=0}^{n-1}\left(\frac{1}{d} \sum_{j=1}^{d} S_{0} \exp \left(\left(r-\sigma^{2} / 2\right) t_{j}+\sigma w_{k, j}\right)-K\right)^{+},
$$

where $\boldsymbol{w}_{k}=\boldsymbol{A} \Phi^{-1}\left(\boldsymbol{t}_{k}\right)$ with lattice point $\boldsymbol{t}_{k}$ for $k=0,1, \ldots, n-1$.

For our particular example we choose the following parameters:

$$
S_{0}=100, \quad r=0.1, \quad \sigma=0.2, \quad T=1, \quad K=100, \quad d=100 .
$$

We choose the principal component analysis method to factorize the matrix $\boldsymbol{\Sigma}$, that is, $\boldsymbol{A}=\left[\sqrt{\lambda_{1}} \boldsymbol{v}_{1}, \cdots, \sqrt{\lambda_{d}} \boldsymbol{v}_{d}\right]$ where $\lambda_{1} \geq \cdots \geq \lambda_{d}$ are the eigenvalues of $\boldsymbol{\Sigma}$ and $\boldsymbol{v}_{1}, \ldots, \boldsymbol{v}_{d}$ are the corresponding unit-length eigenvectors.

In Table 6.2 we present the results obtained with order- 2 weights using 10 random shifts. As a comparison we include also results obtained by the Monte Carlo method (where we also used 10 random shifts). In Fig. 6.4 we plot the graphs of the standard errors obtained by adding one point at a time. (The values of $n$ in both Table 6.2 and Fig. 6.4 correspond to the number of points used from the sequence. Due to the use of 10 random shifts, the actual number of function evaluations in each case is $10 n$.) The results for the order- 2 rule clearly show a rate of convergence close to $\mathcal{O}\left(n^{-1}\right)$ while the Monte Carlo error decays like $\mathcal{O}\left(n^{-1 / 2}\right)$. Furthermore, the graph assures us that it is quite all right to use the embedded lattice rules as a sequence, since the standard error goes down nicely (with some minor zigzag behavior, as expected) as the number of points is increased one at a time. 
TABLE 6.2

Results of the Asian option for Monte Carlo and the order-2 lattice rule

\begin{tabular}{|r|cc|cc|}
\hline$n$ & \multicolumn{2}{|c|}{ order-2 lattice rule } & \multicolumn{2}{c|}{ Monte Carlo } \\
integral & standard error & integral & standard error \\
\hline 1024 & 7.11310 & $5.07 \mathrm{e}-03$ & 7.13816 & $8.97 \mathrm{e}-02$ \\
2048 & 7.10685 & $1.77 \mathrm{e}-03$ & 7.10988 & $5.98 \mathrm{e}-02$ \\
4096 & 7.10252 & $1.56 \mathrm{e}-03$ & 7.12438 & $4.84 \mathrm{e}-02$ \\
8192 & 7.10261 & $6.47 \mathrm{e}-04$ & 7.11989 & $2.54 \mathrm{e}-02$ \\
16384 & 7.10304 & $4.17 \mathrm{e}-04$ & 7.12116 & $2.10 \mathrm{e}-02$ \\
32768 & 7.10324 & $2.34 \mathrm{e}-04$ & 7.12404 & $1.49 \mathrm{e}-02$ \\
65536 & 7.10280 & $1.18 \mathrm{e}-04$ & 7.11410 & $1.14 \mathrm{e}-02$ \\
131072 & 7.10293 & $4.70 \mathrm{e}-05$ & 7.11427 & $9.58 \mathrm{e}-03$ \\
262144 & 7.10287 & $1.91 \mathrm{e}-05$ & 7.10980 & $5.05 \mathrm{e}-03$ \\
524288 & 7.10285 & $1.69 \mathrm{e}-05$ & 7.10636 & $5.00 \mathrm{e}-03$ \\
1048576 & 7.10285 & $8.68 \mathrm{e}-06$ & 7.10709 & $1.69 \mathrm{e}-03$ \\
\hline
\end{tabular}

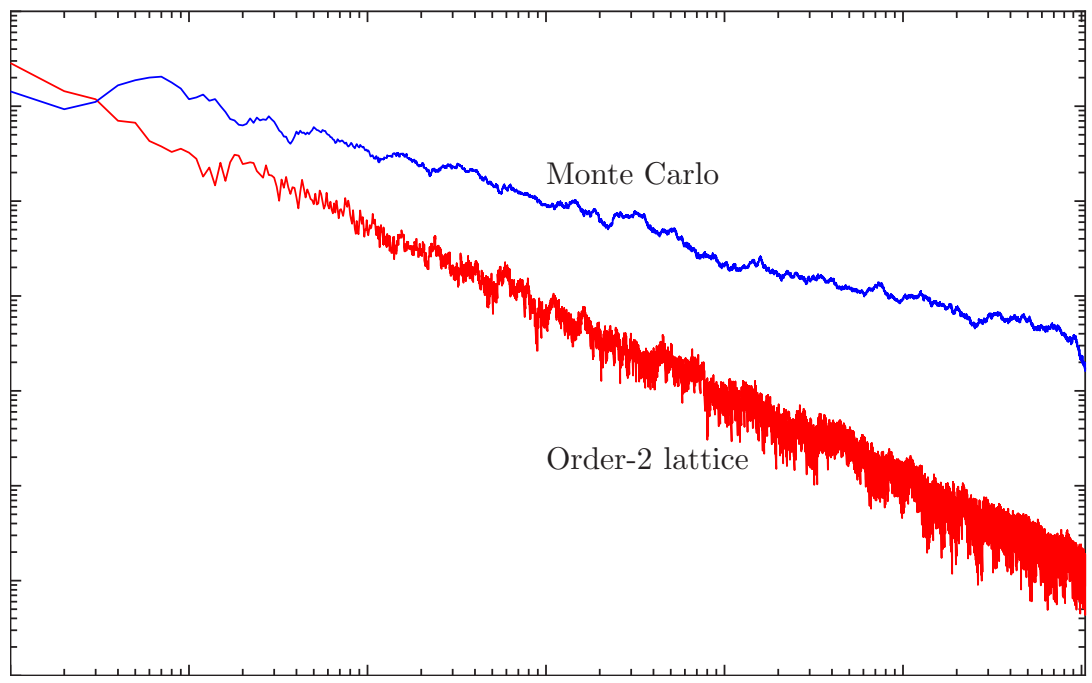

Fig. 6.4. Standard errors of the Asian option for Monte Carlo and the order-2 lattice rule

7. Conclusion. We presented an algorithm for constructing good embedded lattice rules that are extensible in the dimension $d$ and can be used for any number of points $n$ in a pre-defined range. The algorithm utilized both the component-bycomponent construction principle and the fast implementation idea. For product weights and order-dependent weights, the total construction cost is $\mathcal{O}\left(\operatorname{dn}(\log (n))^{2}\right)$ operations, with a linear memory dependence on $n$. Numerical experiments with the unanchored Sobolev space demonstrated that these rules achieve the same optimal rate of convergence $\mathcal{O}\left(n^{-1+\delta}\right), \delta>0$, as those constructed for a fixed number of points, and that the implied constant only gains a factor of 1.30 to 1.55.

Acknowledgment. Financial support from the Onderzoeksfonds K.U.Leuven / Research Fund K.U.Leuven as well as the support of the Australian Research Council under its Centres of Excellence program are gratefully acknowledged. The authors thank Professor Ian Sloan for many helpful comments and rewarding discussions. 


\section{REFERENCES}

[1] M. Abramowitz and I. A. Stegun, eds., Handbook of Mathematical Functions with Formulas, Graphs and Mathematical Tables, vol. 55 of National Bureau of Standards Applied Mathematics Series, U.S. Government Printing Office, Washington, D.C., 1964.

[2] P. P. Boyle, Y. Lai, and K. S. Tan, Pricing options using lattice rules, North Amer. Act. J., (2005), pp. 50-76.

[3] R. E. Caflisch, W. Morokoff, and A. Owen, Valuation of mortgage backed securities using Brownian bridges to reduce effective dimension, J. Comput. Finance, (1997), pp. 27-46.

[4] J. Dick, On the convergence rate of the component-by-component construction of good lattice rules, J. Complexity, 20 (2004), pp. 493-522.

[5] J. Dick, I. H. Sloan, X. Wang, And H. Woźniakowski, Good lattice rules in weighted Korobov spaces with general weights, Numer. Math. To appear. http://www .maths. unsw.edu.au/applied/files/2003/amr03_24.pdf.

[6] F. J. Hickernell, Lattice rules: How well do they measure up?, in Random and Quasi-Random Point Sets, P. Hellekalek and G. Larcher, eds., Springer-Verlag, 1998, pp. 109-166.

[7] F. J. Hickernell, H. S. Hong, P. L'Écuyer, and C. Lemieux, Extensible lattice sequences for quasi-Monte Carlo quadrature, SIAM J. Sci. Comput., 22 (2000), pp. 1117-1138.

[8] F. J. Hickernell And H. Niederreiter, The existence of good extensible rank-1 lattices, J. Complexity, 19 (2003), pp. 286-300.

[9] C. Joy, P. P. Boyle, AND K. S. TAn, Quasi-Monte Carlo methods in numerical finance, Management Science, (1996), pp. 926-938.

[10] F. Y. KUO, Component-by-component constructions achieve the optimal rate of convergence for multivariate integration in weighted Korobov and Sobolev spaces, J. Complexity, 19 (2003), pp. 301-320.

[11] C. Lemieux And P. L'ÉCUYer, Efficiency improvement by lattice rules for pricing Asian options, in Proceedings of the 30th conference on Winter simulation, D. J. Medeiros, E. F. Watson, J. Carson, and M. Manivannan, eds., IEEE, 1998, pp. 579-586.

[12] H. NiederReiter, Random Number Generation and Quasi-Monte Carlo Methods, no. 63 in Regional Conference Series in Applied Mathematics, SIAM, 1992.

[13] D. Nuyens And R. Cools, Fast algorithms for component-by-component construction of rank1 lattice rules in shift-invariant reproducing kernel Hilbert spaces, Math. Comp., 75 (2006), pp. 903-920.

[14] - Fast component-by-component construction of rank-1 lattice rules with a non-prime number of points, J. Complexity, 22 (2006), pp. 4-28.

[15] C. M. RADER, Discrete Fourier transforms when the number of data samples is prime, Proc. IEEE, 5 (1968), pp. 1107-1108.

[16] I. H. Sloan, F. Y. KuO, And S. Joe, Constructing randomly shifted lattice rules in weighted Sobolev spaces, SIAM J. Numer. Anal., 40 (2002), pp. 1650-1665.

[17] I. H. SloAn, X. WANG, AND H. WoźNiakowski, Finite-order weights imply tractability of multivariate integration, J. Complexity, 20 (2004), pp. 46-74.

[18] C. F. VAN LOAN, Computational Frameworks for the Fast Fourier Transform, vol. 10 of Frontiers in Applied Mathematics, SIAM, 1992.

[19] X. WANG AND K. T. FAng, The effective dimension and quasi-Monte Carlo integration, J. Complexity, 19 (2003), pp. 101-124. 
Appendix. Order-2 embedded lattice rule for $2^{10} \leq n \leq 2^{20}$.

\begin{tabular}{|c|c|c|c|c|c|c|c|c|c|c|c|}
\hline$d$ & $z_{d}$ & $d$ & $z_{d}$ & $d$ & $z_{d}$ & $d$ & $z_{d}$ & $d$ & $z_{d}$ & $d$ & $z_{d}$ \\
\hline 1 & 1 & 61 & 390955 & 121 & 151423 & 181 & 455605 & 241 & 8601 & 301 & 83785 \\
\hline 2 & 182667 & 62 & 202915 & 122 & 492123 & 182 & 227317 & 242 & 118219 & 302 & 11217 \\
\hline 3 & 302247 & 63 & 230815 & 123 & 166279 & 183 & 251285 & 243 & 503827 & 303 & 163315 \\
\hline 4 & 433461 & 64 & 304301 & 124 & 335461 & 184 & 295275 & 244 & 368993 & 304 & 293397 \\
\hline 5 & 160317 & 65 & 452315 & 125 & 295093 & 185 & 476155 & 245 & 439439 & 305 & 386597 \\
\hline 6 & 94461 & 66 & 383101 & 126 & 98821 & 186 & 291155 & 246 & 153211 & 306 & 338761 \\
\hline 7 & 481331 & 67 & 70519 & 127 & 118331 & 187 & 294443 & 247 & 282303 & 307 & 88653 \\
\hline 8 & 252345 & 68 & 346393 & 128 & 371123 & 188 & 31913 & 248 & 252999 & 308 & 337581 \\
\hline 9 & 358305 & 69 & 291605 & 129 & 166909 & 189 & 518445 & 249 & 28617 & 309 & 230703 \\
\hline 10 & 221771 & 70 & 397773 & 130 & 369553 & 190 & 481917 & 250 & 514327 & 310 & 140519 \\
\hline 11 & 48157 & 71 & 92713 & 131 & 310959 & 191 & 326981 & 251 & 422201 & 311 & 421913 \\
\hline 12 & 489023 & 72 & 391775 & 132 & 130595 & 192 & 488711 & 252 & 465011 & 312 & 416151 \\
\hline 13 & 438503 & 73 & 131613 & 133 & 417025 & 193 & 447541 & 253 & 160907 & 313 & 197025 \\
\hline 14 & 399693 & 74 & 74351 & 134 & 264103 & 194 & 39629 & 254 & 191687 & 314 & 350607 \\
\hline 15 & 200585 & 75 & 382127 & 135 & 453203 & 195 & 394681 & 255 & 226205 & 315 & 262579 \\
\hline 16 & 169833 & 76 & 219343 & 136 & 497985 & 196 & 379411 & 256 & 254941 & 316 & 510879 \\
\hline 17 & 308325 & 77 & 297125 & 137 & 200509 & 197 & 335309 & 257 & 480375 & 317 & 451713 \\
\hline 18 & 247437 & 78 & 88545 & 138 & 269743 & 198 & 93541 & 258 & 322857 & 318 & 8619 \\
\hline 19 & 281713 & 79 & 89837 & 139 & 461919 & 199 & 188491 & 259 & 456621 & 319 & 50451 \\
\hline 20 & 424209 & 80 & 191863 & 140 & 45927 & 200 & 152371 & 260 & 446175 & 320 & 193793 \\
\hline 21 & 244841 & 81 & 506647 & 141 & 394663 & 201 & 408829 & 261 & 109049 & 321 & 155739 \\
\hline 22 & 205461 & 82 & 441649 & 142 & 299155 & 202 & 217957 & 262 & 285305 & 322 & 23611 \\
\hline 23 & 336811 & 83 & 240063 & 143 & 81299 & 203 & 65145 & 263 & 436731 & 323 & 437689 \\
\hline 24 & 359375 & 84 & 239067 & 144 & 112403 & 204 & 462013 & 264 & 290763 & 324 & 100267 \\
\hline 25 & 86263 & 85 & 310875 & 145 & 447473 & 205 & 220627 & 265 & 290439 & 325 & 439671 \\
\hline 26 & 370621 & 86 & 211625 & 146 & 480325 & 206 & 368989 & 266 & 25363 & 326 & 211341 \\
\hline 27 & 422443 & 87 & 147791 & 147 & 105053 & 207 & 273585 & 267 & 480371 & 327 & 22951 \\
\hline 28 & 284811 & 88 & 370849 & 148 & 328455 & 208 & 373297 & 268 & 153337 & 328 & 73405 \\
\hline 29 & 231547 & 89 & 149445 & 149 & 513239 & 209 & 285793 & 269 & 406899 & 329 & 220037 \\
\hline 30 & 360239 & 90 & 340329 & 150 & 322199 & 210 & 405807 & 270 & 90863 & 330 & 169733 \\
\hline 31 & 505287 & 91 & 493031 & 151 & 47537 & 211 & 63693 & 271 & 78537 & 331 & 408633 \\
\hline 32 & 355195 & 92 & 336897 & 152 & 485183 & 212 & 382573 & 272 & 358757 & 332 & 64171 \\
\hline 33 & 52937 & 93 & 202595 & 153 & 490687 & 213 & 84291 & 273 & 69087 & 333 & 44141 \\
\hline 34 & 344561 & 94 & 518247 & 154 & 214311 & 214 & 444801 & 274 & 431749 & 334 & 122149 \\
\hline 35 & 286935 & 95 & 369599 & 155 & 61871 & 215 & 226471 & 275 & 384083 & 335 & 258491 \\
\hline 36 & 312429 & 96 & 50453 & 156 & 359761 & 216 & 166195 & 276 & 472419 & 336 & 215021 \\
\hline 37 & 513879 & 97 & 133655 & 157 & 509981 & 217 & 170689 & 277 & 298375 & 337 & 11507 \\
\hline 38 & 171905 & 98 & 395941 & 158 & 192829 & 218 & 368423 & 278 & 291499 & 338 & 295341 \\
\hline 39 & 50603 & 99 & 13871 & 159 & 17075 & 219 & 509169 & 279 & 187231 & 339 & 41981 \\
\hline 40 & 441451 & 100 & 248123 & 160 & 486463 & 220 & 243221 & 280 & 6967 & 340 & 378867 \\
\hline 41 & 164379 & 101 & 380725 & 161 & 463461 & 221 & 191447 & 281 & 338357 & 341 & 261545 \\
\hline 42 & 139609 & 102 & 314879 & 162 & 438237 & 222 & 200867 & 282 & 411965 & 342 & 56423 \\
\hline 43 & 371213 & 103 & 106903 & 163 & 444353 & 223 & 194633 & 283 & 447817 & 343 & 445605 \\
\hline 44 & 152351 & 104 & 94505 & 164 & 381279 & 224 & 226469 & 284 & 135463 & 344 & 362017 \\
\hline 45 & 138607 & 105 & 499197 & 165 & 425405 & 225 & 413665 & 285 & 156061 & 345 & 504897 \\
\hline 46 & 441127 & 106 & 479671 & 166 & 179723 & 226 & 148007 & 286 & 356943 & 346 & 335763 \\
\hline 47 & 157037 & 107 & 76553 & 167 & 203897 & 227 & 284505 & 287 & 224431 & 347 & 417407 \\
\hline 48 & 510073 & 108 & 351609 & 168 & 300411 & 228 & 459795 & 288 & 452203 & 348 & 58033 \\
\hline 49 & 281681 & 109 & 13815 & 169 & 35087 & 229 & 324557 & 289 & 284195 & 349 & 80239 \\
\hline 50 & 380297 & 110 & 342069 & 170 & 501519 & 230 & 422149 & 290 & 489071 & 350 & 372353 \\
\hline 51 & 208143 & 111 & 446599 & 171 & 172275 & 231 & 368087 & 291 & 299537 & 351 & 116163 \\
\hline 52 & 497641 & 112 & 374429 & 172 & 278507 & 232 & 166133 & 292 & 313173 & 352 & 287617 \\
\hline 53 & 482925 & 113 & 342011 & 173 & 473739 & 233 & 401441 & 293 & 436265 & 353 & 483795 \\
\hline 54 & 233389 & 114 & 8365 & 174 & 141429 & 234 & 44077 & 294 & 316447 & 354 & 310473 \\
\hline 55 & 238553 & 115 & 424079 & 175 & 117025 & 235 & 457535 & 295 & 8353 & 355 & 75779 \\
\hline 56 & 121499 & 116 & 184381 & 176 & 516465 & 236 & 122611 & 296 & 512723 & 356 & 141943 \\
\hline 57 & 137783 & 117 & 203853 & 177 & 204743 & 237 & 27489 & 297 & 522699 & 357 & 418435 \\
\hline 58 & 463115 & 118 & 7937 & 178 & 505099 & 238 & 392389 & 298 & 453557 & 358 & 305609 \\
\hline 59 & 168681 & 119 & 302301 & 179 & 359937 & 239 & 327231 & 299 & 512829 & 359 & 152901 \\
\hline 60 & 70699 & 120 & 444747 & 180 & 359503 & 240 & 87841 & 300 & 214315 & 360 & 129869 \\
\hline
\end{tabular}

